\title{
Disordered backgammon model
}

\author{
L. Leuzzi ${ }^{1, *}$ and F. Ritort ${ }^{2,3, \dagger}$ \\ ${ }^{1}$ Instituut voor Theoretische Fysica, Universiteit van Amsterdam and FOM, Valckenierstraat 65, 1018 XE Amsterdam, The Netherlands \\ ${ }^{2}$ Departament de Física Fonamental, Facultat de Física, Universitat de Barcelona, Diagonal 647, 08028 Barcelona, Spain \\ ${ }^{3}$ LPTHE et Université Pierre et Marie Curie, Paris VI, Université Denis Diderot, Paris VII, Boite 126, Tour 16, $1^{\text {er }}$ étage, \\ 4 place Jussieu, F-75252 Paris Cedex 05, France
}

(Received 20 December 2001; published 20 May 2002)

\begin{abstract}
In this paper we consider an exactly solvable model that displays glassy behavior at zero temperature due to entropic barriers. The new ingredient of the model is the existence of different energy scales or modes associated with different relaxational time scales. Low-temperature relaxation takes place by partial equilibration of successive lower-energy modes. An adiabatic scaling solution, defined in terms of a threshold energy scale $\epsilon^{*}$, is proposed. For such a solution, modes with energy $\epsilon \gg \epsilon^{*}$ are equilibrated at the bath temperature, modes with $\epsilon \ll \epsilon^{*}$ remain out of equilibrium, and relaxation occurs in the neighborhood of the threshold $\epsilon$ $\sim \epsilon^{*}$. The model is presented as a toy example to investigate the conditions related to the existence of an effective temperature in glassy systems and its possible dependence on the energy sector is probed by the corresponding observable.
\end{abstract}

DOI: 10.1103/PhysRevE.65.056125

PACS number(s): 05.70.-a, 61.43.Bn, 61.43.Fs

\section{INTRODUCTION}

The study of exactly solvable models has always been an active area of research in the field of statistical physics. They help us to grasp the general principles governing the physical behavior of realistic systems, which, due to the complicated interactions among the different constituents, cannot be predicted using standard perturbative techniques. Glasses, in general, are systems falling into this category. The slow relaxation of glasses observed in the laboratory is a consequence of the simultaneous interplay of its constituents, which yields a very complex and rich phenomenology.

It is well known that glasses fall out of equilibrium when the characteristic observation time is larger than their relaxation time. Because the relaxation time is strongly dependent on temperature, it turns out that glasses are immediately out of equilibrium as soon as the temperature is few degrees below the glass transition. Well below the glass transition temperature $T_{g}$ no time evolution is apparently observed in the glass and one is tempted to conclude that the glass is in a stationary state. Nothing more far from the truth. Glasses still relax but slow enough for any change to be observable in laboratory time scales. Old experiments on polymers reveal that the slowly relaxing state corresponds to an aging state [1]. That is, if the system is perturbed while being in its aging state, then the characteristic time associated with the response of the system scales with the age of the system (i.e., the time elapsed since it was quenched). Another way to look at this aging phenomena is to evaluate the time autocorrelation function. It is observed that the typical decorrelation time scales with the age of the system [2].

A simple scenario to explain these results is the following. Consider a liquid well above $T_{g}$ where correlations decay exponentially with time. One may consider the resultant be-

\footnotetext{
*Email address: leuzzi@wins.uva.nl

${ }^{\dagger}$ Email address: ritort@ffn.ub.es
}

havior of the liquid as the superposition of different and independent harmonic modes. Each of these energy modes corresponds to a normal mode of a system and describes a collective oscillation of $N$ atoms around their local minima. This is the harmonic approximation that is known to work quite well in liquids. Nevertheless, already as the temperature goes below a critical temperature $T_{c}$ (the transition temperature predicted by the mode-coupling theory [3]) other collective modes different from that of the standard vibrational ones become important. The nature of these modes is quite different from the usual harmonic normal modes because they do not represent oscillations around a given configuration within a metastable well but transitions among different wells. These modes are reminiscent of some type of instanton solutions recently computed in the framework of some spin glass models [4]. Below $T_{c}$, relaxational dynamics proceeds by activation over the barriers characterizing these collective modes. Now, the main difference between these collective modes and the usual harmonic modes relies on how they relax to equilibrium when put in contact with a thermal bath at temperature $T$. Relaxation to equilibrium is determined by the height of the energy barriers separating different modes. Suppose a given normal mode has frequency $\omega_{k}$ and energy $E_{k} \propto \omega_{k}^{2}$. The relaxation time for each of these modes is typically of order $\tau_{k} \sim \exp \left(E_{k} / k_{B} T\right)$. Therefore, as the energy $E_{k}$ becomes lower than the thermal bath temperature this mode rapidly equilibrates. On the contrary, if $E_{k} \gg k_{B} T$, this mode remains frozen. Collective modes are different. As the reference energy of the collective modes depletes, the typical barrier separating these modes increases leading to the contrary behavior and to superactivation effects. While high-energy collective modes are separated by low barriers, low-energy collective modes are separated by high barriers. A simple schematic representation of this scenario in a one-dimensional configurational space is shown in Fig. 1. This behavior is common to the majority of exactly solvable glassy models $[5-7,18]$, phenomenological trap 


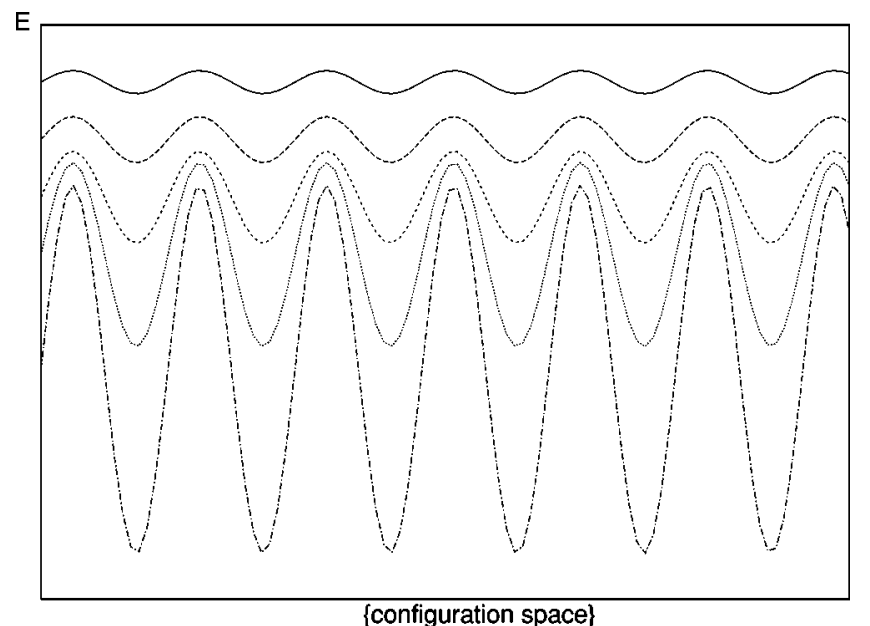

FIG. 1. A one-dimensional example of an energy landscape of collective modes: high-energy collective modes are separated by low barriers while low-energy collective modes are separated by high barriers.

models [8], and kinetically constrained models [9] to cite a few.

In what follows we will use the generic word mode to refer to this kind of collective excitation. Let us label the modes with the integer variable $r$ and let us denote their energy by $\epsilon_{r}$. Let us suppose that the energy levels are ordered from lower to higher energies according to the label $r$. It is natural to assume that there is a characteristic mode $r^{*}$ with associated energy $\epsilon^{*}$ such that, all modes with $\epsilon_{r} \gg \epsilon^{*}$ have already relaxed, while in the other limit, $\epsilon_{r} \ll \epsilon^{*}$, all modes are frozen. If the system is quenched well below $T_{c}$ then equilibration cannot be achieved in laboratory time scales, this means that all modes below $\epsilon^{*}$ remain frozen while modes above $\epsilon^{*}$ remain equilibrated at the bath temperature. The energy threshold $\epsilon^{*}$ decays with time because, as time goes by, higher barriers are accessible to the system. The resulting scenario is that of a liquid where collective modes above $\epsilon^{*}$ are in some sort of local equilibrium at the temperature of the bath $T$ while modes below $\epsilon^{*}$ are frozen. This scenario, as it stands, is too naive because it is based on the assumption that there are no dynamical correlations between the different modes, i.e., the high-energy modes do not influence the low-energy modes. While this is true in the equilibrium state it may not be valid (and indeed it is not) when any type of local dynamics induces correlations between different modes. Still it is interesting to investigate in which conditions such a description turns out to be correct. Examples where this type of description holds are mean field models [5,10-13]. By mean-field we mean those models where there is no spatial dimensionality associated with the set of interactions. In these cases, different modes are not related to different length scales. Therefore local dynamical rules do not necessarily induce correlations between the different modes. Generally speaking, the identification of this energy scale remains an open problem for which we do not have yet a complete understanding.

This energy scale $\epsilon^{*}$ is related to what has received the name of effective or fictive temperature $T_{\text {eff }}$ in the most re- cent literature about glassy dynamics [14-20]. $T_{\text {eff }}$ is an effective time-dependent parameter describing equilibrium fluctuations for those thermalized modes with $\epsilon \gg \epsilon^{*}$ $=k_{B} T_{\text {eff }}$. While a thermometer coupled to the fast modes is expected to measure the bath temperature, a thermometer coupled to the slower ones should yield the effective temperature. Hence, the effective temperature corresponds to an energy threshold that separates collective modes which are frozen from those which have relaxed to the bath temperature. Relaxation, then, takes place at energy scales in the neighborhood of the threshold value $\epsilon^{*}$. For real systems, this energy threshold is related to the typical volume of the drop that is able to release strain energy during its relaxation to equilibrium. A scenario on this type of physical mechanism for glassy relaxation has recently been introduced in Ref. [21] where this threshold energy $\epsilon^{*}$ has been related to the size of the cooperative region as an explanation of the fragility and superactivation anomalies in real glasses.

The existence on an effective temperature is tightly related to the validity of some approximations used in the context of slowly relaxing systems such as the adiabatic approximation. Within the adiabatic approximation one obtains a Markovian description for the dynamics. Again, this Markovian description encodes within a single parameter (the effective temperature) all the complicated past of the system. The adiabatic approximation has been shown to give the correct asymptotic dynamical behavior in some simple models of glasses such as the backgammon model [22,23] while for the majority of the other most famous models in the literature (for instance the $p$-spin model [5]) the implementation of such approximation is generally not yet known. Quite probably, a Markovian description for glassy dynamics is unrealistic and the original idea of experimentalists [24] to encode the dynamical behavior into effective parameters such as the effective pressure or the effective (else said fictive) temperature could be the only labels without a deep physical meaning.

These ideas have been contrasted in several exactly solvable models. All these models have the advantage of being mean field, hence dynamical equations can be closed in one or another way. Still, the mean-field character of these models does not generally allow to investigate in a simple way the relaxational properties of the different modes of the spectrum. In general, the dynamics of all these models has been studied by addressing the closure of the dynamics associated with the global quantities (such as the energy or correlationresponse functions) that do not discern the contribution to the global relaxation of the different energy modes. Examples of these models are the $p$-spin model $[5,10,13,16]$, the backgammon [20,22,23,25], the harmonic oscillator, and other spherical spin models $[7,16-18]$. The question of the existence of an effective temperature is also tightly related to the particular way in which the fluctuation-dissipation theorem (FDT) is violated $[13,26,27]$. The question whether there exists a single effective temperature describing the violation of FDT is still controversial (see, for instance, Ref. [28]). We believe that this and other related questions can be better addressed, even at the level of mean-field models, by analyzing the contribution of all different modes. The analysis of 
the harmonic vibrational modes in glasses is already an interesting step in this direction [29] although here we have in mind other type of collective modes.

The purpose of this paper is the study of a model where the dynamical relaxation of the different energy modes can be made explicitly clear. This model is what we refer to as the disordered backgammon model (DBG model) and consists in a generalization of the backgammon model to allow different energies for different boxes. Again, just like its predecessor, the slow relaxation of this model is due to entropic barriers. For the DBG model we show the existence of an energy threshold $\epsilon^{*}(t)$ that separates the equilibrated modes from the nonequilibrated modes. The DBG model [with its associated threshold $\epsilon^{*}(t)$ ] provides a microscopic realization that is reminiscent of some phenomenological models proposed in the past such as the trap model in a tree considered by Bouchaud and Dean [30]. The advantage in the DBG model is that now one can exhaustively investigate the distinct relaxation of each of the different energy modes verifying whether the scenario of the effective temperature presented before holds. This will help to better understand the meaning of the effective temperature and its relation with the violation fluctuation-dissipation theorem.

In Sec. II we introduce the DBG model. Its thermodynamic properties are reported in Sec. III, where the special case of very low temperatures is explicitly worked out. In Sec. IV we present the dynamical equations whose solutions are found within an adiabatic approximation in Sec. V. There the features of such an approximation are carefully analyzed. In Sec. VI numerical results are presented for two specific models belonging to the family of DBG models. Finally, in Sec. VII, a method is introduced in order to estimate the threshold energy scale $\epsilon^{*}$ directly from the dynamics. In Sec. VIII we present our conclusions. Some technical issues are presented in four different appendixes.

\section{THE DISORDERED BACKGAMMON MODEL}

\section{A. Definition of the model}

Let us take $N$ particles that can occupy $N$ boxes, each one labeled by an index $r$ that runs from 1 to $N$. Suppose now that all particles are distributed among the boxes. A given box $r$ contributes to the Hamiltonian with an energy $-\epsilon_{r}$ only when it is empty. In this case the total Hamiltonian of the system reads

$$
\mathcal{H}=-\sum_{r=1}^{N} \epsilon_{r} \delta_{n_{r}, 0},
$$

where $\delta$ is the Kronecker delta and $n_{r}$ denotes the occupancy or number of particles in box $r$. The $\epsilon_{r}$ are quenched random variables extracted from a distribution $g(\epsilon)$ that we assume to be defined only for $\epsilon \geqslant 0$. The interest of this definition will be discussed below. Like in the original backgammon (BG) model [6] we consider Monte Carlo mean-field dynamics where a particle is randomly chosen in a departure box and a move to an arrival box $a$ is proposed. If $d$ denotes the departure box, the proposed change is accepted according to the Metropolis rule with probability $W(\Delta E)$ $=\min [1, \exp (-\beta \Delta E)]$ where $\Delta E=\epsilon_{a} \delta_{n_{a}, 0}-\epsilon_{d} \delta_{n_{d}, 1}$. Note that the departure box satisfies $n_{d} \geqslant 1$ and departure boxes are chosen with probability $n_{d} / N$. This dynamics corresponds to the Maxwell statistics where the particles are distinguishable and differs from the one corresponding to the Bose statistics $[31,23,32,20]$ where the particles are indistinguishable and arrival boxes are chosen with uniform probability $1 / N$.

In the dynamics the total number of particles is conserved so that the occupancies satisfy the closure condition

$$
\sum_{r=1}^{N} n_{r}=N
$$

Now we want to show that the interesting case corresponds to the situation where $g(\epsilon)$ is only defined for $\epsilon \geqslant 0$. In this case, the dynamics turns out to be extremely slow at low temperatures, similarly to what happens for the original BG model. The difference lies in the type of ground state. The ground state of Eq. (1) corresponds to the case where all particles occupy a single box, the one with the smallest value of $\epsilon$. Let us denote by $\epsilon_{0}$ this smallest value. Then the ground state energy is given by the relation $E_{G S}=$ $-\sum_{r=1}^{N} \epsilon_{r}+\epsilon_{0}$. Since all the $\epsilon$ are positive no other configuration can have a lower energy. If $g(\epsilon)$ is a continuous distribution the ground state is also unique. Now it is easy to understand that, during the dynamical evolution at zero temperature, all boxes with high values of $\epsilon$ become empty quite soon and the dynamics involves boxes with progressively lower values of $\epsilon$. The asymptotic dynamics is then determined by the behavior of the distribution $g(\epsilon)$ in the limit $\epsilon \rightarrow 0$. If $g(\epsilon) \sim \epsilon^{\alpha}$, for $\epsilon \rightarrow 0$, we will show that the asymptotic long-time properties only depend on $\alpha$. Note that the normalization of the $g(\epsilon)$ imposes $\alpha>-1$. This classification includes also the original BG model where there is no disorder at all. In that case $g(\epsilon)=\delta(\epsilon-1)$ so the distribution has a finite gap at $\epsilon=0$. The behavior corresponding to this singular energy distribution can be obtained from the previous one in the limiting case $\alpha \rightarrow \infty$.

One important aspect of the model (1) is that, in the presence of disorder, it is not invariant under an arbitrary constant shift of the energy levels. Actually, by changing $\epsilon_{r}$ $\rightarrow \epsilon_{r}^{\prime}=\epsilon_{r}+c$ with $c \geqslant 0$, the model turns out to be a combination of the original model and the classical BG model. After shifting, the new distribution $g\left(\epsilon^{\prime}-c\right)$ has a finite gap (equal to $c$ plus the gap of the original distribution). The new model corresponds again to the $\alpha \rightarrow \infty$ case and the asymptotic dynamical behavior coincides with that of the standard BG model. As we will see later, the present model is characterized by an energy threshold $\epsilon^{*}$ that drives the relaxation to the stationary state. Only when the energy threshold can go to zero we have a different asymptotic behavior. For all models with a finite gap, $\epsilon^{*}$ cannot be smaller than the gap, hence asymptotically sticks to the gap and the relaxational behavior of the DBG model with a finite gap corresponds to that of the standard BG model.

One of the outstanding features of this model is that a description of the dynamics in the framework of an adiabatic approximation turns out to be totally independent from the 
type of distribution $g(\epsilon)$ (and hence of $\alpha$ ) despite the fact that the asymptotic long-time behavior of the effective temperature and of the internal energy depends on the value of $\alpha$.

\section{B. Observables}

Like in the original BG model we define the occupation probabilities, $P_{k}$, that a box contains $k$ particle,

$$
P_{k}=\frac{1}{N} \sum_{r=1}^{N} \delta_{n_{r}, k}
$$

and the corresponding densities that a box of energy $\epsilon$ contains $k$ particles

$$
\begin{gathered}
g_{k}(\epsilon)=\frac{1}{N} \sum_{r=1}^{N} \delta\left(\epsilon_{r}-\epsilon\right) \delta_{n_{r}, k}, \quad k \geqslant 0, \\
g(\epsilon)=\frac{1}{N} \sum_{r=1}^{N} \delta\left(\epsilon_{r}-\epsilon\right) .
\end{gathered}
$$

The $P_{k}$ and the $g_{k}$ are related by

$$
P_{k}=\int_{0}^{\infty} g_{k}(\epsilon) d \epsilon, \quad k \geqslant 0,
$$

and the conservation of particles reads

$$
\sum_{k=0}^{\infty} P_{k}=1, \quad \sum_{k=0}^{\infty} g_{k}(\boldsymbol{\epsilon})=\frac{g(\boldsymbol{\epsilon})}{\int_{0}^{\infty} g(\boldsymbol{\epsilon}) d \boldsymbol{\epsilon}}=g(\boldsymbol{\epsilon}) .
$$

The energy can be expressed in terms of the density $g_{0}(\epsilon)$ as

$$
E=-\int_{0}^{\infty} d \epsilon \epsilon g_{0}(\epsilon)
$$

This set of observables depends on time through the time evolution of the occupancies $n_{r}$ of all boxes. In the following section we analyze the main equilibrium properties of the model.

\section{EQUILIBRIUM BEHAVIOR}

The solution of the thermodynamics proceeds similarly as for the case of the original BG model. The partition function can be computed in the grand partition ensemble. It reads

$$
\mathcal{Z}_{G C}=\sum_{N=0}^{\infty} \mathcal{Z}_{C}(N) z^{N}
$$

where $z=\exp (\beta \mu)$ is the fugacity, $\mu$ is the chemical potential, and $\mathcal{Z}_{C}(N)$ stands for the canonical partition function of a system with $N$ particles. The canonical partition function can be written as

$$
\mathcal{Z}_{C}=\sum_{n_{r}=0}^{N} \frac{N !}{\prod_{r=1}^{N} n_{r} !} \exp \left(\beta \sum_{r=1}^{N} \epsilon_{r} \delta_{n_{r}, 0}\right) \delta_{N,} \sum_{r=1}^{N} n_{r},
$$

where $\delta_{i, j}$ is the Kronecker delta. Introducing this expression in Eq. (9) we can write $\mathcal{Z}_{G C}$ as an unrestricted sum for all the occupancies $n_{r}$,

$$
\mathcal{Z}_{G C}=N ! \sum_{n_{r}=0}^{\infty} \prod_{r=1}^{N} \frac{z^{n_{r}}}{n_{r} !} \exp \left(\beta \epsilon_{r} \delta_{n_{r}, 0}\right) .
$$

The factor $N ! / \Pi_{r} n_{r}$ ! in the partition function $\mathcal{Z}_{C}$ is introduced to account for the distinguishability of particles. This factor leads to an overextensive entropy (i.e., the Gibbs paradox) that can be cured eliminating from $\mathcal{Z}_{G C}$ the overcounting term $N$ ! in the numerator. The final result is

$$
\mathcal{Z}_{G C}=\exp \left\{\sum_{r=1}^{N} \ln \left[\sum_{n=0}^{\infty} \frac{z^{n}}{n !} \exp \left(\beta \epsilon_{r} \delta_{n, 0}\right)\right]\right\},
$$

yielding the grand-canonical potential energy per box

$$
\begin{aligned}
G & =F-\mu=-T \frac{\ln \left(\mathcal{Z}_{G C}\right)}{N} \\
& =-T \sum_{r=1}^{N} \ln \left[\exp \left(\beta \epsilon_{r}\right)+\exp (z)-1\right] \\
& =-T \int_{0}^{\infty} g(\epsilon) \ln [\exp (\beta \epsilon)+\exp (z)-1] d \epsilon,
\end{aligned}
$$

where $F$ is the Helmholtz free energy per box. The fugacity $z$ is determined by the conservation condition (2) that reads

$$
\frac{\partial G}{\partial \mu}=-1 \quad \text { or, } \quad \text { equivalently, } \quad \frac{\partial F}{\partial \mu}=0,
$$

yielding the closure condition

$$
\int_{0}^{\infty} \frac{g(\epsilon)}{\exp (\beta \epsilon)+\exp (z)-1} d \epsilon=\frac{1}{z \exp (z)} .
$$

This equation gives the fugacity $z$ as function of $\beta$ and, from Eq. (13) and its derivatives, the whole thermodynamics. In particular, the equilibrium expressions for $g_{k}(\epsilon)$ are

$$
g_{k}^{\mathrm{eq}}(\boldsymbol{\epsilon})=\frac{z^{k} g(\boldsymbol{\epsilon}) \exp \left(\beta \epsilon \delta_{k, 0}\right)}{k ![\exp (\beta \epsilon)+\exp (z)-1]},
$$

the corresponding $P_{k}$ being given by Eq. (6), which together with the closure relation (15), leads to the expression

$$
P_{k}^{\mathrm{eq}}=\delta_{k, 0}\left(1-\frac{\exp (z)-1}{z \exp (z)}\right)+\left(1-\delta_{k, 0}\right) \frac{z^{k-1}}{k ! \exp (z)} \text {. }
$$

Starting from Eq. (8) the equilibrium energy density is obtained as 


$$
E^{\mathrm{eq}}=-\int_{0}^{\infty} d \epsilon \frac{\epsilon g(\boldsymbol{\epsilon}) \exp (\beta \boldsymbol{\epsilon})}{\exp (\beta \boldsymbol{\epsilon})+\exp (z)-1} .
$$

All these expressions can be evaluated at finite temperature. Note that, although the values of $P_{k}^{\mathrm{eq}}$ in Eq. (17) are independent of the disorder distribution $g(\epsilon)$, they directly depend on that distribution through the equilibrium value of $z$ [which obviously depends on the $g(\epsilon)]$. Of particular interest for the dynamical behavior of the model are the lowtemperature properties that we analyze below.

\section{Thermodynamics at low temperatures}

A perturbative expansion can be carried out close to $T$ $\rightarrow 0$ to find the leading behavior of different thermodynamic quantities. Let us start analyzing the closure condition (15). Doing the transformation $\epsilon^{\prime}=\beta \epsilon$, Eq. (15) can be rewritten as

$$
T \int_{0}^{\infty} \frac{g\left(T \epsilon^{\prime}\right)}{\exp \left(\epsilon^{\prime}\right)+\exp (z)-1} d \epsilon^{\prime}=\frac{1}{z \exp (z)} .
$$

In the limit $T \rightarrow 0$ the fugacity $z$ depends on the behavior of $g\left(T \epsilon^{\prime}\right)$ in the limit $T=0$, i.e., on the behavior of $g(\epsilon)$ for $\epsilon \rightarrow 0$. Assuming $g(\epsilon) \sim \epsilon^{\alpha}$ for $\epsilon \ll 1$ we define the function $h(\epsilon)$ through the relation $g(\epsilon)=\epsilon^{\alpha} h(\epsilon)$, where $h(\epsilon)$ is a smooth function of $\epsilon$ with $h(0)$ finite. The integral can be expanded around $T=0$ by taking successive derivatives of the function $h$,

$$
\begin{gathered}
z \exp (z) T^{\alpha+1} \int_{0}^{\infty} \frac{\epsilon^{\prime \alpha}}{\exp \epsilon^{\prime}+\exp (z)-1} \\
\times \sum_{k=0}^{\infty} \frac{h^{(k)}(0)\left(T \epsilon^{\prime}\right)^{k}}{k !} d \epsilon^{\prime}=1 .
\end{gathered}
$$

Using the asymptotic result $z \rightarrow \infty$, when $T \rightarrow 0$, everything reduces to estimate the following integral in the large $z$ limit:

$$
\int_{0}^{\infty} d x \frac{x^{\alpha+k}}{\exp (x)+\exp (z)-1} \sim z^{\alpha+k+1} \exp (-z) .
$$

The term $k=0$ in the series yields the leading behavior for $z$, which turns out to be

$$
z \sim \beta^{(\alpha+1) /(\alpha+2)} .
$$

In a similar way the energy can be computed to leading order in $T$,

$$
E=E_{G S}+a T+O\left(T^{2}\right),
$$

giving a finite specific heat at low temperatures. In Sec. VI we will show explicitly such a behavior for two specific DBG models, one with $\alpha=1$ [defined in Eq. (48), Sec. VI] and the other with $\alpha=0$ [Eq. (49), Sec. VI]. Solving Eq. (15) numerically for $z(T)$ in each specific model and inserting $z(T)$ in the expression (18) for the equilibrium energy density, we get the energy dependence on the temperature. In all cases, at equilibrium, the energy is linear for low $T$, as pre-

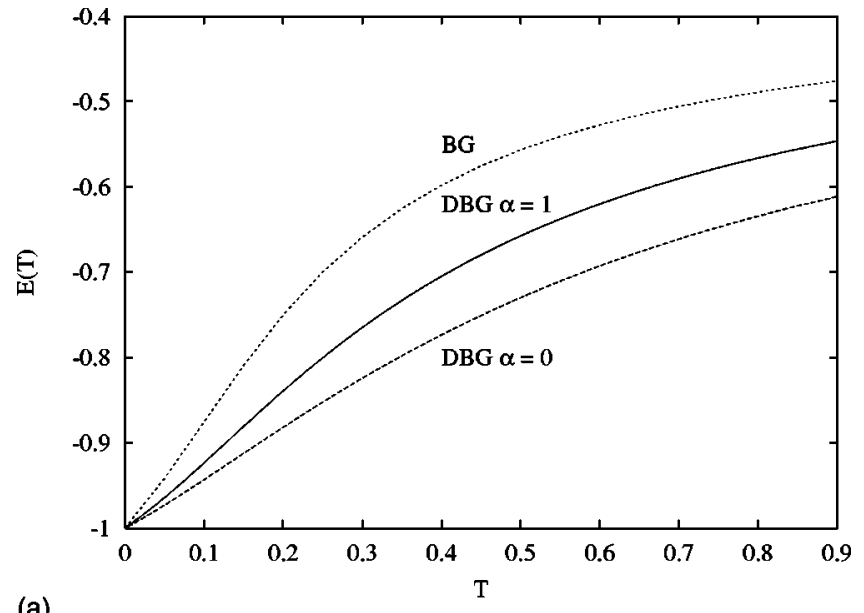

(a)

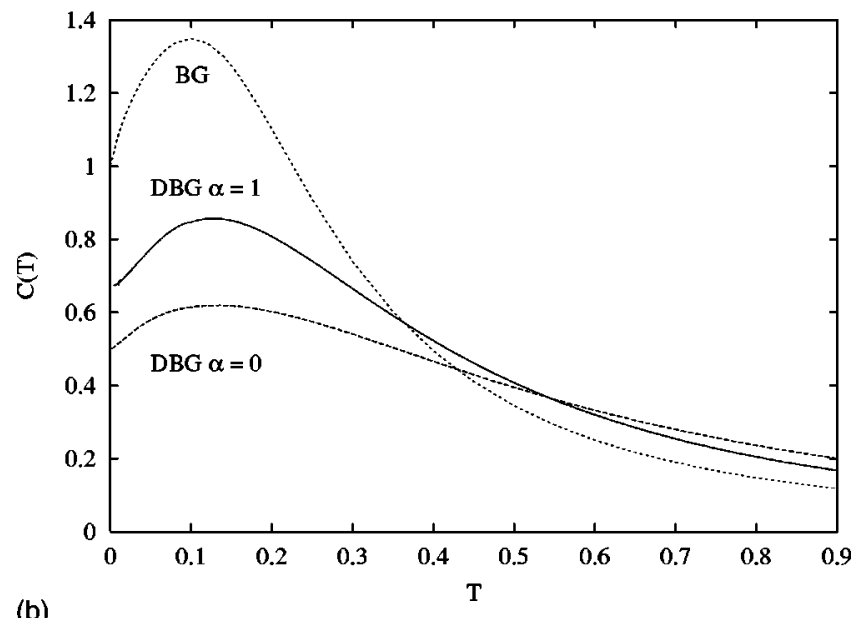

FIG. 2. Left, energy dependence on temperature at equilibrium for two DBG models, with $\alpha=1$ and $\alpha=0$. Also the energy for the standard BG model $(\alpha \rightarrow \infty)$ is plotted. Right, specific heat for the same models. For all cases (disordered or not) it turns out to be finite at $T=0$.

dicted in Eq. (23). It yields, therefore, a finite specific heat as corresponds to a classical model with Maxwell-Boltzmann statistics (see Fig. 2).

\section{DYNAMICAL EQUATIONS}

Here we consider the dynamical equations for the occupation probabilities $P_{k}$ and their associated densities $g_{k}(\epsilon)$. The dynamical equations in this model are derived in a similar way as for the standard BG model. The main difference is that in the DBG the equations for the occupancies probabilities $P_{k}$ do not generate a closed hierarchy of equations. Only for $T=0$ such a closed hierarchy is obtained. As we will see later, this has important consequences when we become interested in the zero-temperature relaxation.

A hierarchy of equations can only be obtained at the level of the occupation probability densities $g_{k}(\epsilon)$. A detailed derivation of these equations is reported in Appendix A. Here we show the final result, 


$$
\begin{aligned}
& \frac{\partial g_{0}(\epsilon)}{\partial t}=g_{1}(\epsilon)\left[1+\int_{\epsilon}^{\infty} d \epsilon^{\prime} g_{0}\left(\epsilon^{\prime}\right)\left(e^{-\beta\left(\epsilon^{\prime}-\epsilon\right)}-1\right)\right]-g_{0}(\epsilon) \\
& \times\left[e^{-\beta \epsilon}+P_{1}\left(1-e^{-\beta \epsilon}\right)\right. \\
& \left.+\int_{0}^{\epsilon} d \epsilon^{\prime} g_{1}\left(\epsilon^{\prime}\right)\left(e^{-\beta\left(\epsilon-\epsilon^{\prime}\right)}-1\right)\right], \quad k=0, \\
& \frac{\partial g_{1}(\epsilon)}{\partial t}=2 g_{2}(\epsilon)\left(1+\int_{0}^{\infty} d \epsilon g_{0}(\epsilon) e^{-\beta \epsilon}-P_{0}\right)-g_{1}(\epsilon) \\
& \times\left[2+\int_{\epsilon}^{\infty} d \epsilon^{\prime} g_{0}\left(\epsilon^{\prime}\right)\left(e^{-\beta\left(\epsilon^{\prime}-\epsilon\right)}-1\right)\right]+g_{0}(\epsilon) \\
& \times\left[e^{-\beta \epsilon}+P_{1}\left(1-e^{-\beta \epsilon}\right)+\int_{0}^{\epsilon} d \epsilon^{\prime} g_{1}\left(\epsilon^{\prime}\right)\right. \\
& \left.\times\left(e^{-\beta\left(\epsilon-\epsilon^{\prime}\right)}-1\right)\right], \quad k=1 \\
& \frac{\partial g_{k}(\boldsymbol{\epsilon})}{\partial t}=(k+1) g_{k+1}(\boldsymbol{\epsilon})\left(1+\int_{0}^{\infty} d \epsilon g_{0}(\boldsymbol{\epsilon}) e^{-\beta \epsilon}-P_{0}\right) \\
& -g_{k}(\epsilon)\left[1+k+k\left(\int_{0}^{\infty} d \epsilon g_{0}(\epsilon) e^{-\beta \epsilon}-P_{0}\right)\right] \\
& +g_{k-1}(\epsilon), \quad k>1 \text {. }
\end{aligned}
$$

The equations for the $P_{k}$ are directly obtained by integrating the $g_{k}(\epsilon)$ according to Eq. (6). The result is

$$
\begin{aligned}
\frac{d P_{k}(t)}{d t}= & (k+1)\left[P_{k+1}(t)-P_{k}(t)\right]+P_{k-1} \\
& +\left(\int_{0}^{\infty} d \epsilon g_{0}(\epsilon) e^{-\beta \epsilon}-P_{0}\right)\left[\delta_{k, 1}-\delta_{k, 0}-k P_{k}(t)\right. \\
& \left.+(k+1) P_{k+1}(t)\right]
\end{aligned}
$$

with $P_{-1}=0$. It is easy to check that the equilibrium solutions (16) are indeed stationary solutions. As previously said, for general $\beta$, the equations for the $P_{k}$ do not generate a hierarchy by themselves but depend on the $g_{k}(\epsilon)$ through the distribution $g_{0}(\epsilon)$ in Eq. (27). Nevertheless, a remarkable aspect is that they generate a well defined hierarchy at $T$ $=0$, which coincides with the equations of the original $\mathrm{BG}$ model [25]. These are

$$
\begin{aligned}
\frac{d P_{k}(t)}{d t}= & (k+1)\left[P_{k+1}(t)-P_{k}(t)\right]+P_{k-1} \\
& -P_{0}\left[\delta_{k, 1}-\delta_{k, 0}-k P_{k}(t)+(k+1) P_{k+1}(t)\right] .
\end{aligned}
$$

It is easy to understand why at $T=0$ the dynamical equations are independent of the density of states $g(\epsilon)$. The reasoning is as follows. For $T=0$, all moves of particles between departure and arrival boxes with different energies $\epsilon_{d}$ and $\epsilon_{a}$ depend on the precise values of these energies only when the departure box contains a single particle and the arrival box is empty. But such a move does not lead to any change in any of the $P_{k}$, hence dynamical equations for the $P_{k}$ remain independent of $g(\epsilon)$. Obviously this does not hold for other observables such as the energy $E$ [i.e., the mean value of $\epsilon$ over the density $\left.g_{0}(\epsilon)\right]$ and higher moments of $g_{k}(\epsilon)$.

This observation is crucial, since we look at the glassy behavior at $T=0$. It turns out that the analysis of the dynamical equations for the DBG at $T=0$ decomposes into two parts. On one hand, the equations for the $P_{k}$ coincide with those of the original nondisordered BG model, while on the other hand, in order to analyze the behavior of the energy one must analyze the behavior of the hierarchy of equations for the $g_{k}(\epsilon)$, which is quite complicated. In the following section we will see how the analysis of these equations can be done within the framework of a generalized adiabatic approximation.

\section{THE ADIABATIC APPROXIMATION}

\section{A. Standard adiabatic solution for the $\boldsymbol{P}_{\boldsymbol{k}}$}

In this section we are interested in the solution of the dynamical equations at $T=0$. We already saw that the Eqs. (28) for the $P_{k}$ coincides with those of the original nondisordered BG model. Consequently, the same adiabatic approximation used for the $P_{k}$ in the original BG model is still valid for the DBG. Let us remind the main results [22,23]. The key idea behind that approximation is that, while $P_{0}$ constitutes a slow mode, the other $P_{k}$ with $k>0$ are fast modes. Hence they can be considered as if they were in equilibrium at the hypersurface in phase space $P_{0}$ $=$ constant, this constant being given by the actual value of $P_{0}$ at time $t$. In the original BG model $P_{0}=-E$, hence thermalization of the fast modes $P_{k}(k>0)$ occurs on the hypersurface of constant energy. For the DBG model this is not true, the hypersurface where equilibration of fast modes occurs does not coincide with the constant energy hypersurface simply because the energy and $P_{0}$ are different quantities. Indeed, we will see later that their leading time behavior is different.

At $T=0$ the equation for $P_{0}$ [Eq. (28) for $\left.k=0\right]$ reads

$$
\frac{\partial P_{0}}{\partial t}=P_{1}\left(1-P_{0}\right) \text {. }
$$

If local equilibrium is reached on the hypersurface of constant $P_{0}$ we can relate $P_{1}$ to $P_{0}$ using Eqs. (17). The simplest way of dealing with Eq. (29) is to relate both $P_{1}$ and $P_{0}$ to the time-dependent fugacity $z^{*}$ writing down a dynamical equation for $z^{*}$. Using Eq. (17) this yields

$$
\frac{\partial z^{*}}{\partial t}=\frac{z^{*}\left[\exp \left(z^{*}\right)-1\right]}{\exp \left(z^{*}\right)\left[\exp \left(z^{*}\right)-z^{*}-1\right]}
$$

In the large time limit $z^{*}$ diverges and the leading asymptotic behavior is given by $z^{*} \simeq \ln (t)+\ln [\ln (t)]$. The oc- 
cupation probabilities are, then, given by the relations (17) replacing $z$ by the time-dependent fugacity $z^{*}$, as long as $z^{*} \gg 1$

$$
P_{k} \simeq \delta_{k, 0}\left(1-\frac{1}{z^{*}}\right)+\left(1-\delta_{k, 0}\right) \frac{\left(z^{*}\right)^{k-1}}{k ! \exp \left(z^{*}\right)} .
$$

Hence, according to Eq. (22), the inverse effective temperature is given by the relation

$$
\beta_{\text {eff }} \sim\left(z^{*}\right)^{(\alpha+2) /(\alpha+1)} \sim[\ln (t)]^{(\alpha+2) /(\alpha+1)}
$$

and the effective temperature depends on the properties of the disorder distribution $g(\epsilon)$ in the limit $\epsilon \rightarrow 0$ through the value of the exponent $\alpha$. Clearly, when the density of levels decreases as we approach $\epsilon=0$, the relaxation turns out to be slower; the limiting case being the original $\mathrm{BG}$ model for which $\alpha \rightarrow \infty$ and $\beta_{\text {eff }} \sim \ln (t)$. In the other limit $\alpha \rightarrow-1$, when disorder becomes unnormalized, the inverse effective temperature diverges very fast. Already from Eq. (23) one can anticipate that the same asymptotic behavior holds for the energy [see Eq. (44)], hence $\alpha$ interpolates between fast relaxation $(\alpha=-1)$ and very slow relaxation $(\alpha=\infty)$. A relaxation slower than logarithmic is not possible in the present model.

\section{B. Generalized adiabatic solution for the $g_{k}(\epsilon)$}

The equations for the $g_{k}(\epsilon)$ at $T=0$ are

$$
\begin{gathered}
\frac{\partial g_{0}(\epsilon)}{\partial t}=g_{1}(\epsilon)\left[1-\int_{\epsilon}^{\infty} d \epsilon^{\prime} g_{0}\left(\epsilon^{\prime}\right)\right] \\
-g_{0}(\epsilon) \int_{\epsilon}^{\infty} d \epsilon^{\prime} g_{1}\left(\epsilon^{\prime}\right), \quad k=0, \\
\frac{\partial g_{1}(\epsilon)}{\partial t}=2 g_{2}(\epsilon)\left(1-P_{0}\right)-g_{1}(\epsilon)\left[2-\int_{\epsilon}^{\infty} d \epsilon^{\prime} g_{0}\left(\epsilon^{\prime}\right)\right] \\
+g_{0}(\epsilon) \int_{\epsilon}^{\infty} d \epsilon^{\prime} g_{1}\left(\epsilon^{\prime}\right), \quad k=1, \\
\frac{\partial g_{k}(\epsilon)}{\partial t}=(k+1) g_{k+1}(\epsilon)\left(1-P_{0}\right)-g_{k}(\epsilon)\left[1+k\left(1-P_{0}\right)\right] \\
+g_{k-1}(\epsilon), \quad k>1 .
\end{gathered}
$$

To solve the dynamical equations for the $g_{k}(\epsilon)$ in the adiabatic approximation we note that, contrarily to the global quantities $P_{k}$, they cannot be equilibrated among all different modes. The reason is that, due to the entropic character of the relaxation, very low energy modes are rarely involved because the time needed to empty one further box increases progressively as time goes by, hence they cannot be thought as effectively thermalized. Note that in the original BG model all boxes have the same energy, hence there is a unique class of modes. For the general disordered model we expect the existence of a time dependent energy scale $\epsilon^{*}$ separating the equilibrated and the nonequilibrated modes.
The mechanism of relaxation is the one we reminded in the Introduction when speaking about the behavior of collective modes. At zero temperature there is no thermal activation and the equilibrated modes are in the sector $\epsilon \gg \epsilon^{*}$ while the nonequilibrated modes are in the other sector $\epsilon \ll \epsilon^{*}$. The value of $\epsilon^{*}$ can be easily guessed. After quenching to zero temperature the system starts to relax to its ground state. Because there is no thermal activation, relaxation is driven by entropic barriers, i.e., flat directions in configurational space through which the system diffuses. Entropic relaxation is energy costless, so its rate is determined by the number of available configurations with energy smaller than or equal to the actual energy. A simple microcanonical argument giving the relaxation rate goes as follows. Let us denote by $M$ the number of occupied boxes and by $\Omega(M)$ the number of configurations with $M$ occupied boxes. The typical time to increase by unity the number of empty boxes is given by

$$
\tau \simeq \frac{\Omega(M)}{\Omega(M-1)} .
$$

For distinguishable particles we have $\Omega(M) \simeq M^{N}$. In the large $N, M$ limit we get

$$
\tau \simeq\left(\frac{M}{M-1}\right)^{N} \simeq \exp \left(\frac{N}{M}\right) .
$$

Using the relation $P_{0}=1-M / N$ and Eq. (36) we find

$$
\frac{d P_{0}}{d t}=-\frac{\Delta M}{N \Delta t}=\frac{1}{\tau}=\exp \left(-\frac{1}{1-P_{0}}\right),
$$

where we have used the fact that at zero temperature the number of occupied boxes can only decrease by unity, thus $\Delta M=-1$.

This yields the result $P_{0} \simeq 1-1 /\{\ln (t)+\ln [\ln (t)]\}$ in agreement with the adiabatic approximation. Actually, from the solution in Eq. (30) for $z^{*}$ and using the adiabatic relation (31), we obtain $P_{0} \simeq 1-1 / z^{*}$, which yields the same result. The typical relaxation time (37) behaves, then, like $\tau$ $\simeq \exp \left[1 /\left(1-P_{0}\right)\right] \simeq \exp \left(z^{*}\right)$.

If the threshold $\epsilon^{*}$ plays the role of an energy barrier and $\beta_{\text {eff }}$ accounts for the effective thermal activation due to entropic effects, we obtain, for the typical relaxation time, $\tau \simeq \exp \left(\beta_{\mathrm{eff}} \epsilon^{*}\right)$. This expression is only valid to the leading order. As we will see below there are subleading corrections to this expression arising for the fact that the relaxation time is better described by the expression $\tau$ $\simeq \exp \left(\beta_{\text {eff }} \epsilon^{*}\right) /\left(\beta_{\text {eff }} \epsilon^{*}\right)$ [see Eq. (45)]. Hence, at a given time scale $t$ (i.e., the time elapsed since the system was quenched) all modes where $\tau \ll t$ are equilibrated at zero temperature (which in this case is the temperature of the thermal bath) and therefore frozen. Modes with $\tau \gg t$, although dynamically evolving, are also frozen because the barriers (in this case entropic barriers) are too high to allow for relaxation within the time scale $t$.

Only those modes whose characteristic time is $\tau \sim t$ are relaxing at a given time scale $t$. We get for the time depen- 
dent energy scale $\epsilon^{*}$ and the effective temperature the relation $\epsilon^{*} \sim \ln (t) / \beta_{\text {eff }}$ and this yields the leading behavior

$$
\epsilon^{*} \sim[\ln (t)]^{-1 /(\alpha+1)} .
$$

According to what has been said one can impose the following ansatz solution for the $g_{k}(\epsilon)$. If $g_{k}^{\mathrm{eq}}$ stands for the equilibrium density at $T=0$ [i.e., according to Eq. (16), $g_{k}^{\text {eq }}$ $\left.=g(\epsilon) \delta_{k, 0}\right]$ then we have

$$
\Delta g_{k}(\epsilon) \equiv g_{k}(\epsilon)-g_{k}^{\mathrm{eq}}(\epsilon)=\frac{\Delta P_{k}}{\epsilon^{*}} \hat{g}_{k}\left(\frac{\epsilon}{\epsilon^{*}}\right),
$$

where $\Delta P_{k} \equiv P_{k}-P_{k}^{\mathrm{eq}}=P_{k}-\delta_{k, 0}$ and $\hat{g}_{k}(x)$ decays pretty fast to zero for $x>1$. This expression tells us the following. Above $\epsilon^{*}$ the $g_{k}(\epsilon)$ have relaxed to their corresponding equilibrium distributions at the temperature of the bath (in this case the bath is at zero temperature). On the other hand, in the sector of the energy spectrum where $\epsilon<\epsilon^{*}$, the densities $g_{k}$ are still relaxing [specially in the region $\epsilon / \epsilon^{*}$ $\sim O(1)]$. Since the relaxation is driven by the shift in time of the threshold energy $\epsilon^{*}$ the proposed scaling solution ansatz seems quite reasonable. The prefactor $\Delta P_{k} / \epsilon^{*}$ is introduced to fulfill condition (6). Furthermore, the condition $\int_{0}^{\infty} d x \hat{g}_{k}(x)=1$ is imposed on the scaling function $\hat{g}_{k}$.

In Appendix B we show how this ansatz closes the set of equations (33) reproducing also the leading asymptotic behavior for $\epsilon^{*}$ and $z^{*}$, which turns out to be

$$
\begin{gathered}
\epsilon^{*} \simeq \frac{1}{[\ln (t)]^{1 /(\alpha+1)}}, \\
z^{*} \simeq \frac{1}{\left(\epsilon^{*}\right)^{1+\alpha}} \simeq \ln (t) .
\end{gathered}
$$

For later use, we define the following function:

$$
G_{k}(\epsilon) \equiv \frac{\Delta g_{k}(\epsilon) \epsilon^{*}}{\Delta P_{k}}=\hat{g}_{k}\left(\frac{\epsilon}{\epsilon^{*}}\right),
$$

which scales as function of $\epsilon / \epsilon^{*}$. The scaling relation (40) yields the leading asymptotic behavior of all observables different from the occupation probabilities $P_{k}$. For instance, the energy is given by $E=-\int_{0}^{\infty} d \epsilon \epsilon g_{0}(\epsilon)$; using the scaling relation (40) and the asymptotic expression (41) we get for the leading term

$$
\begin{aligned}
E-E_{G S} & \sim-\int_{0}^{\infty} d \epsilon\left[g_{0}(\epsilon)-g(\epsilon)\right] \epsilon \sim\left(\epsilon^{*}\right)^{\alpha+2} \\
& \sim \frac{1}{\ln (t)^{(\alpha+2) /(\alpha+1)}} .
\end{aligned}
$$

Note that the asymptotic scaling behavior of the energy is the same as for the effective temperature $T_{\text {eff }}=1 / \beta_{\text {eff }}$ in agreement with the quasiequilibrium hypothesis [see Eq. (23)]. An important result is that the threshold $\epsilon^{*}$ decays slower to zero than the effective temperature. A case where this difference can be clearly appreciated corresponds to the case where the density of states vanishes exponentially fast $g(\epsilon) \sim \exp (-A / \epsilon)$. In this case, $\epsilon^{*}$ decays slower than logarithmically, namely, like $1 / \ln [\ln (t)]$ (see Appendix B for details).

\section{Relaxational spectrum in equilibrium}

One of the crucial features behind the applicability of the adiabatic approximation is that the long-time behavior at zero temperature finds its correspondence with the lowtemperature relaxational properties of the equilibrium state.

To analyze the spectrum of relaxation times $\tau_{\mathrm{eq}}(\epsilon)$ in equilibrium we expand up to first order in perturbation theory the dynamical equations for the $g_{k}(\epsilon)$ around their equilibrium solutions $g_{k}^{\mathrm{eq}}(\epsilon)$. Using the expansion $g_{k}(\epsilon)$ $=g_{k}^{\mathrm{eq}}(\epsilon)+\delta g_{k}(\epsilon)$ we get a set of equations for the variations $\delta g_{k}(\epsilon)$. These are shown in Appendix C.

A complete derivation of the relaxation time $\tau(\epsilon)$ in equilibrium is complicated. But it is easy to convince oneself that the relaxation time is asymptotically (in the limit $T \rightarrow 0$ ) strongly peaked around the threshold energy $\epsilon^{*}$. For $\epsilon \gg \epsilon^{*}$ the relaxation time is small because the population of high energy boxes in equilibrium is rather small. On the other hand, for $\epsilon / \epsilon^{*} \ll 1$ the relaxation is estimated to be finite and independent of $T$. This result is derived in the aforementioned Appendix $\mathrm{C}$ where we show that the maximum relaxation time occurs for $\epsilon$ around $\epsilon^{*}$. Starting from Eqs. (C1) for $\delta g_{0}(\epsilon)$ and $\delta g_{1}(\epsilon)$ and making use of the adiabatic Ansatz (40), we find, for $\epsilon \simeq \epsilon^{*}$,

$$
\tau\left(\epsilon^{*}\right) \sim \frac{e^{\beta \epsilon^{*}}}{\beta \epsilon^{*}},
$$

where $\epsilon^{*}(T) \sim T^{1 /(2+\alpha)}$, is the asymptotic temperature dependence of the threshold energy at low temperature. This yields for the temperature dependence of the relaxation time,

$$
\tau(T) \sim \frac{e^{\beta^{(\alpha+1) /(\alpha+2)}}}{\beta^{(\alpha+1) /(\alpha+2)}}
$$

showing that there is an activated behavior as a function of the temperature but with a relaxation time that increases slower than Arrhenius as $T \rightarrow 0$. Note that for the standard BG model corresponding to $\alpha \rightarrow \infty$ we obtain an Arrhenius behavior and in the opposite limit $\alpha \rightarrow-1$ the relaxation time does not diverge anymore.

\section{NUMERICAL RESULTS}

In this section we numerically check the main results obtained in the previous sections. In particular, we want to show the existence of the threshold energy $\epsilon^{*}$ separating the equilibrated modes from the nonequilibrated energy modes. We have compared three different models characterized by three different types of distributions (Fig. 3). All three distributions were chosen to satisfy the conditions 


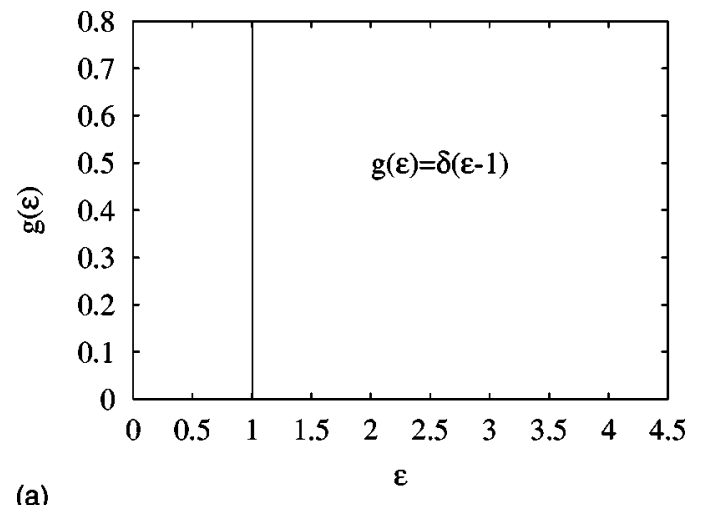

(a)

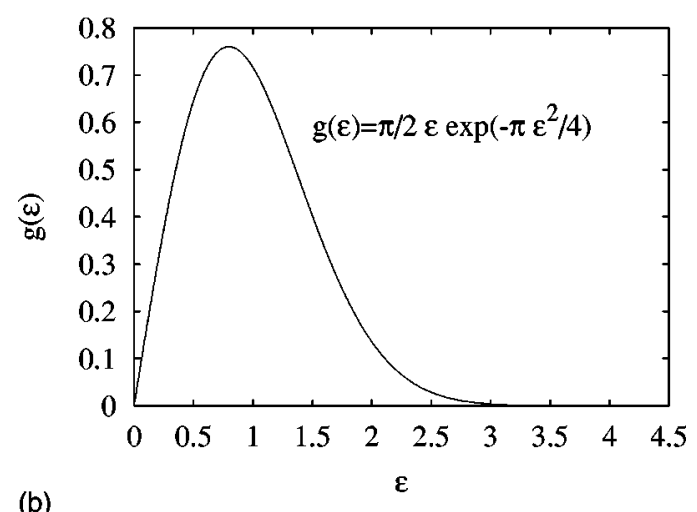

(b)

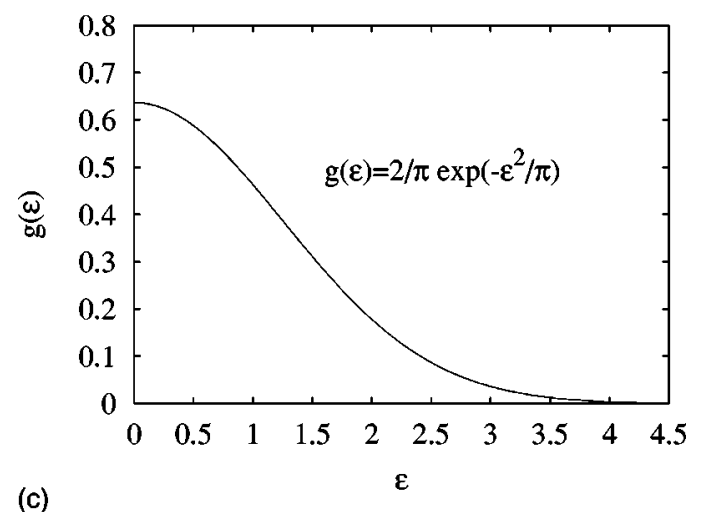

(c)

FIG. 3. Probability distribution of the energy weights of the boxes of the DBG model. (a) The standard backgammon model has no disordered distribution, all boxes have the same weight. (b) The probability distribution function of a DBG with $\alpha=1$, at very low energy the density of boxes goes to zero. (c) A second DBG model with $\alpha=0$. Here the probability of having boxes with energies arbitrarily close to zero is finite.

$$
\int_{0}^{\infty} d \epsilon g(\epsilon)=\int_{0}^{\infty} d \epsilon \epsilon g(\epsilon)=1,
$$

in such a way that the ground state has energy $E_{G S}=-1$ in the limit $N \rightarrow \infty$ for all three cases. The models are the following ones.

Case A. Nondisordered model with a gap [Fig. 3(a)]. This is the original BG model where $g(\epsilon)=\delta(\epsilon-1)$. This case corresponds to $\alpha \rightarrow \infty$, therefore $\epsilon^{*}=1$ and the threshold energy is time independent. The energy is expected to decay

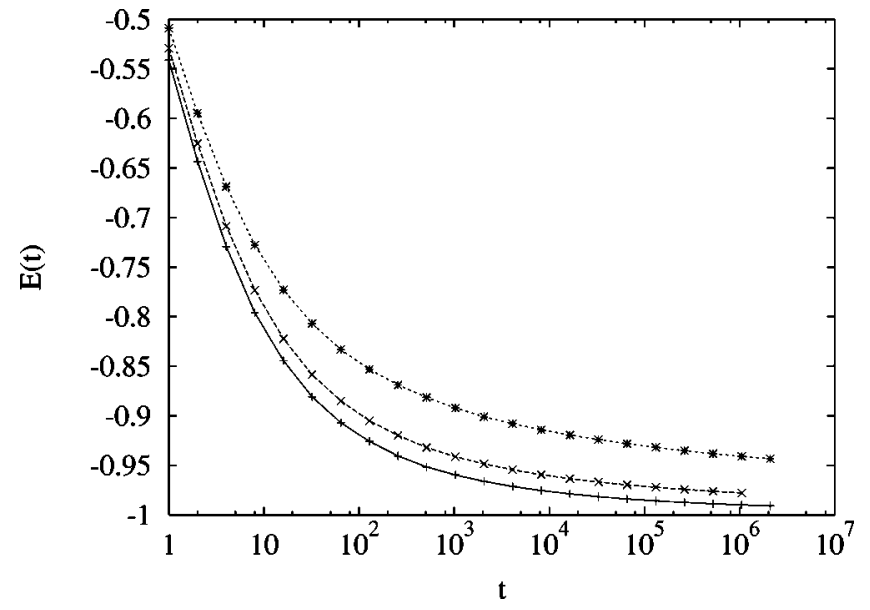

FIG. 4. Energy as a function of time for the three models discussed in the text. The energy was computed averaging over time intervals $\Delta t=2^{k}, k$ being an integer. The lower curve represents the relaxation in the $\alpha=0$ DBG model (case C), the middle curve the $\alpha=1$ DBG model (case B), and the upper curve the standard BG model (case A).

like $E+1 \sim T_{\text {eff }} \sim 1 / \ln (t)$. As previously said in Sec. II, the same behavior is expected for any disorder distribution $g(\epsilon)$ with a finite gap.

Case B. Disordered model without a gap but $g(0)=0[$ Fig. 3(b)]. We have considered the distribution

$$
g(\epsilon)=\frac{\pi}{2} \epsilon \exp \left(-\frac{\pi}{4} \epsilon^{2}\right)
$$

This case corresponds to $\alpha=1$. The energy threshold $\epsilon^{*}$ scales like $1 / \sqrt{\ln (t)}$ and the effective temperature and the energy scale like $E+1 \sim T_{\text {eff }} \sim 1 /[\ln (t)]^{3 / 2}$.

Case $C$. Disordered model without a gap and $g(0)$ finite [Fig. 3(c)]. We have considered the distribution

$$
g(\epsilon)=\frac{2}{\pi} \exp \left(-\frac{\epsilon^{2}}{\pi}\right) .
$$

This case corresponds to $\alpha=0$. The energy threshold $\epsilon^{*}$ scales like $1 / \ln (t)$ and the effective temperature and the energy scale like $E+1 \sim T_{\text {eff }} \sim 1 /[\ln (t)]^{2}$.

In Fig. 4 we plot the decay of the energy for all three models. Simulations were done for $N=10^{4}, 10^{5}, 10^{6}$ boxes (the number of particles is identical to the number of boxes) showing that finite-size effects are not big in the asymptotic regime. We show data for one sample and $N=10^{6}$. We plot the energy as a function of time starting from a random initial condition [particles randomly distributed among boxes, $E(t=0)=-1 / e]$. As clearly seen from the figure, relaxation is faster for the case $\mathrm{C}$ and slower for the standard $\mathrm{BG}$ model (case A).

The different asymptotic behaviors are shown in Fig. 5 . There we plot $\left[E(t)-E_{G S}\right][\ln (t)]^{\lambda}$ with $\lambda \equiv(\alpha+2) /(\alpha+1)$. To avoid finite-size corrections when the energy is close to its ground state we computed exactly $E_{G S}$ $=(1 / N)\left(-\sum_{r=1}^{N} \epsilon_{r}+\epsilon_{\min }\right)$ where $\epsilon_{\min }$ is the minimum value 


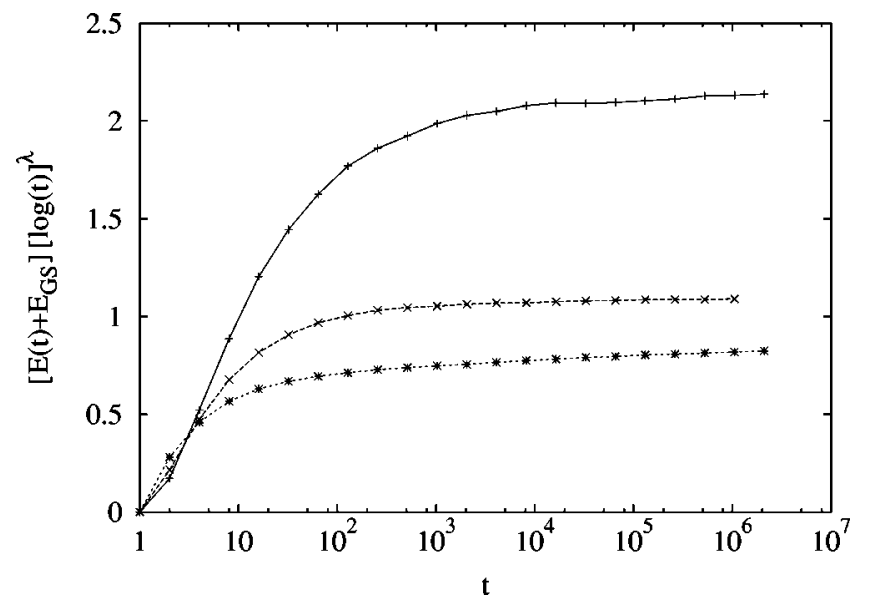

FIG. 5. $\left(E-E_{G S}\right)[\ln (t)]^{\lambda}$ plotted as function of time with $\lambda$ $=(\alpha+2) /(\alpha+1)$ for the three different models discussed in the text. The upper curve refers to case $\mathrm{C}$, the middle one to case $\mathrm{B}$, and the lower one to standard BG model (case A).

among the $\epsilon^{\prime} s$. The different curves saturate at a finite quantity corresponding to the asymptotic leading constant. Note that the convergence is slow, showing the presence of subleading logarithmic corrections to the leading behavior.

Let us now analyze the shape of the probability densities $g_{k}(\epsilon)$. For this distribution we also only show results for $N$ $=10^{6}$ because a smaller number of boxes results in higher noise in the curves. The distribution probabilities were numerically computed by binning the $\epsilon$ axis from $\epsilon=0$ up to $\epsilon=\epsilon_{\max }$ where $\epsilon_{\max }$ is the maximum value of $\epsilon_{r}$ among all the $N$ boxes. One hundred bins are enough to see the behavior of the time evolution of the different distributions. In Figs. 6 and 7 we show the $g_{0}(\epsilon)$ for cases B and C, respectively. Note that the $g_{0}(\epsilon)$ converge to the asymptotic result $g(\epsilon)$ for $\epsilon>\epsilon^{*}$ in agreement with the adiabatic solution (40) while they are clearly different for $\epsilon<\epsilon^{*}$. The value of $\epsilon^{*}$ where $g_{0}(\epsilon)$ deviates from the asymptotic curve $g(\epsilon)$ shifts slowly to zero [like $1 /(\ln (t))^{1 / 2}$ or $1 / \ln (t)$ for cases B and C, respectively], as can be seen in Figs. 6 and 7 .

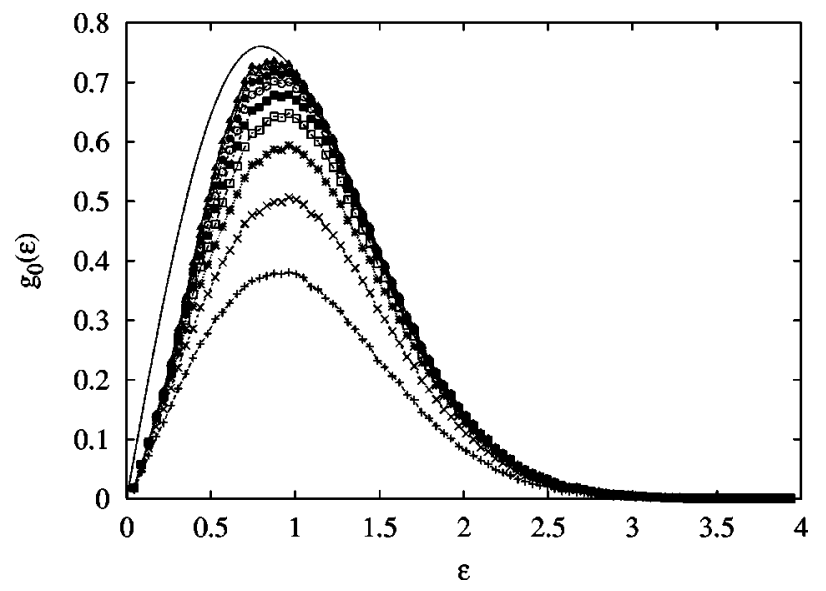

FIG. 6. Distribution $g_{0}(\epsilon)$ for case B for different times $2^{k}$ with $k=4,6,8,10,12,14,16,18,20$ (from bottom to top). The continuous line is $g(\epsilon)$ given in Eq. (48).

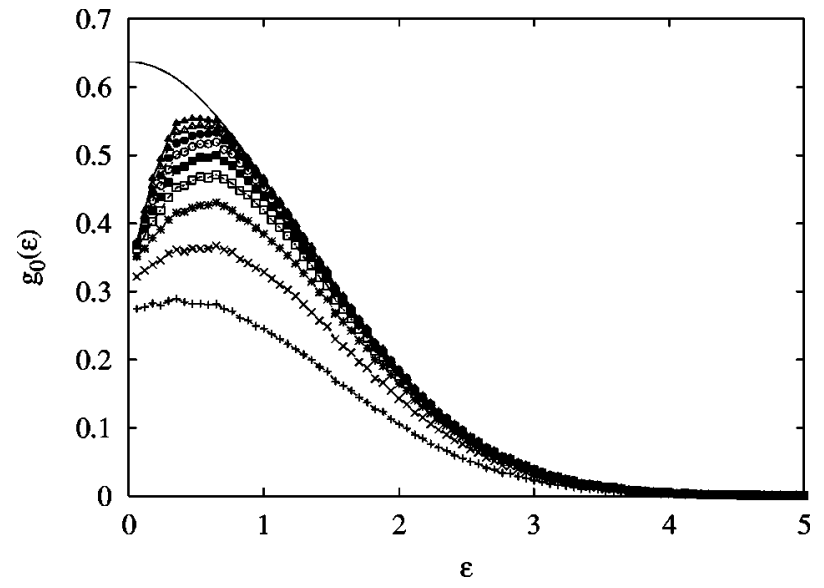

FIG. 7. Distribution $g_{0}(\epsilon)$ for case $\mathrm{C}$ for different times $2^{k}$ with $k=4,6,8,10,12,14,16,18,20$ (from bottom to top). The continuous line is $g(\epsilon)$ given in Eq. (49).

We do not show results for the other $g_{k}$ (for instance, $g_{1}$ ) because they decay very fast to zero (already for $t=2^{17}$ there are no occupied boxes with more than one particle). Instead, in Figs. 8 and 9 we verify the adiabatic ansatz, Eqs. (40) and (43), for the densities $g_{0}$ and $g_{1}$ in the two models B and C. Figure 8 plots $G_{0}(\epsilon)$ for both models. Figure 9 plots $G_{1}(\epsilon)$ for both models. We have used the relation (39) together with $z^{*}=\ln (t)+\ln [\ln (t)]$ yielding

$$
\begin{gathered}
G_{0}(\epsilon)=\frac{\Delta g_{0}(\epsilon)\{\ln (t)+\ln [\ln (t)]\}}{\ln (t)^{1 /(\alpha+1)}}=\hat{g}_{0}\left(\frac{\epsilon}{\epsilon^{*}}\right), \\
G_{1}(\epsilon)=\frac{\Delta g_{1}(\epsilon) t}{\ln (t)^{\alpha /(\alpha+1)}}=\hat{g}_{1}\left(\frac{\epsilon}{\epsilon^{*}}\right) .
\end{gathered}
$$

Note that the scaling is pretty well satisfied and that the $\hat{g}_{k}(x)$ indeed vanishes for $x \simeq 1$ yielding an estimate for $\epsilon^{*}$ is both cases. We find, $\epsilon^{*} \simeq 6 / \sqrt{\ln (t)}$ for case $B$ and $\epsilon^{*}$

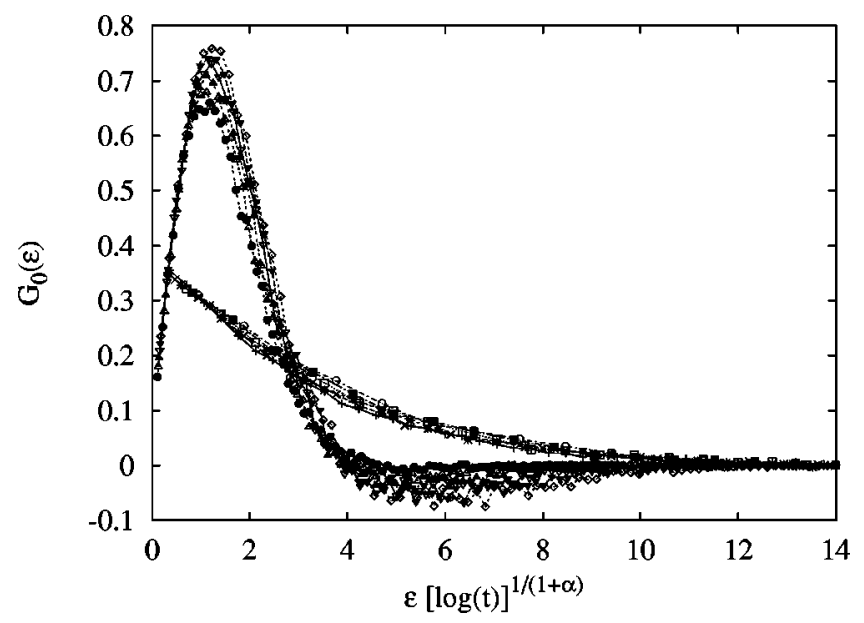

FIG. 8. Distribution $G_{0}(\epsilon)$ as a function of $\epsilon \sqrt{\ln (t)}$ for case B (distribution with a maximum) and as a function of $\epsilon \ln (t)$ for case $\mathrm{C}$ (monotonically decreasing distribution). Times are $t=2^{k}$ with $k$ $=6,8,10,12,14,16$. 


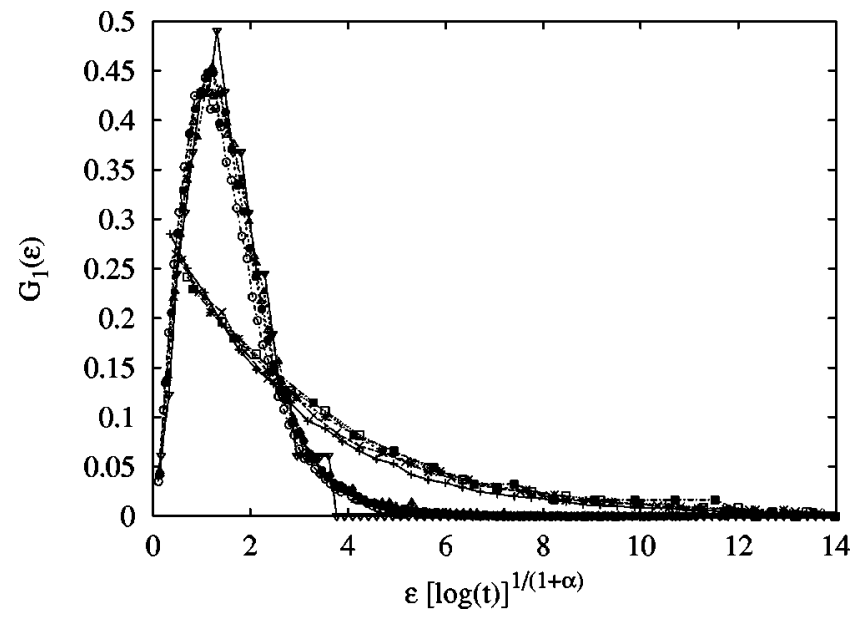

FIG. 9. Distribution $G_{1}(\epsilon)$ as a function of $\epsilon \sqrt{\ln (t)}$ for case B (distribution with a maximum) and as a function of $\epsilon \ln (t)$ for case $\mathrm{C}$ (monotonically decreasing distribution). Times are $t=2^{k}$ with $k$ $=6,8,10,12,14$.

$\simeq 12 / \ln (t)$ for case C. Note also that the quality of the collapse of the $G_{0}$ is slightly worse for case B than for case C. We think that this is due to the stronger subleading corrections to the shift of $\epsilon^{*}$, which decays slower to zero for case B. Hence the asymptotic regime is reached only for later times. Indeed, as Fig. 6 shows, the value of $\epsilon^{*}$ obtained within our time scales has not yet reached the maximum of the distribution $g(\epsilon)$, so that we are still far from the asymptotic behavior $g\left(\epsilon^{*}\right) \sim \epsilon^{*}$. Yet, it is remarkable how well the scaling ansatz Eqs. (40) and (43) fits the numerical data.

\section{A METHOD TO DETERMINE THE THRESHOLD ENERGY SCALE $\epsilon^{*}$}

In this section we are interested in the following question. Is there a general method to determine the energy scale $\epsilon^{*}$ without having any precise information about the adiabatic modes present in the system? In the previous sections we addressed this question by proposing an adiabatic scaling ansatz to the dynamical equations. Here we propose a general method to determine the energy scale $\epsilon^{*}$ from first principles without the necessity of knowing the nature of the slow modes present in the system. Obviously for models such as the standard BG model this energy scale has no role since we know from the beginning that the relaxation takes place on a single energy scale.

Consider the following quantity $P(\Delta E)$ defined as the normalized probability density of having a first accepted energy change $\Delta E$ at time $t$. Let us consider the case of zero temperature where this probability density is defined only for $\Delta E \leqslant 0$. If $Q(\Delta E)$ denotes the probability of proposing an energy change at time $t$ (the move is not necessarily accepted), it is easy to show that $P$ and $Q$ are proportional to each other,

$$
P(\Delta E)=\frac{Q(\Delta E)}{A} \theta(-\Delta E),
$$

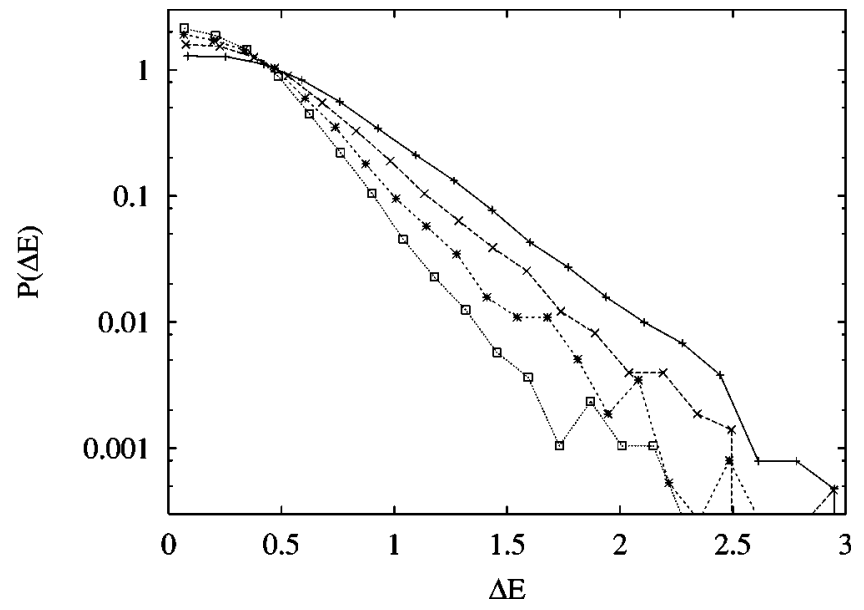

FIG. 10. $P(\Delta E)$ versus $\Delta E$ for different times $t$ $=10^{2}, 10^{3}, 10^{4}, 10^{5}$ (from top to bottom) computed as explained in the text.

where $A=\int_{-\infty}^{0} Q(\Delta E) d \Delta E$ is the acceptance rate. The expression for $Q(\Delta E)$ [and therefore $P(\Delta E)$ ] can be exactly computed. Note that computing $Q(\Delta E)$ yields all information about the statistics of energy changes, in particular the evolution equation for the energy. ${ }^{1}$ On the contrary, given the time evolution for the energy this does not necessarily yield the distribution $Q(\Delta E)$. For the DBG this function can be exactly derived (its derivation is shown in Appendix D). Here we quote the result,

$$
\begin{aligned}
P(\Delta E)= & \frac{\int_{0}^{\infty} d \epsilon g_{0}(\epsilon) g_{1}(\epsilon-\Delta E)+\left(1-P_{0}\right) g_{1}(-\Delta E)}{A} \\
& \times \theta(-\Delta E),
\end{aligned}
$$

with

$$
A=\int_{0}^{\infty} d \epsilon^{\prime} \int_{\epsilon^{\prime}}^{\infty} d \epsilon g_{0}\left(\epsilon^{\prime}\right) g_{1}(\epsilon)+\left(1-P_{0}\right) P_{1} .
$$

Using the scaling ansatz Eq. (40) we obtain the simple scaling scaling relation,

$$
P(\Delta E)=\frac{1}{\epsilon^{*}} \hat{P}\left(\frac{\Delta E}{\epsilon^{*}}\right) .
$$

A collapse of different $P(\Delta E)$ for different times can be used to determine the time evolution of $\epsilon^{*}$. In Fig. 10 we show the scaling of $P(\Delta E)$ for the model B for $N=10^{4}$ and different times $t=10^{2}, 10^{3}, 10^{4}, 10^{5}$. Starting from a random initial configuration, statistics has been collected over approximately 30000 jumps for every time. In Fig. 11 we check the scaling relation (55) plotting $P(\Delta E) \epsilon^{*}$ as a function of $\Delta E / \epsilon^{*}$ where we have taken $\epsilon^{*} \sim 1 / \sqrt{\ln (t)}$. Note also

\footnotetext{
${ }^{1}$ Actually, in equilibrium at finite temperature $Q(\Delta E)$ satisfies detailed balance $Q(\Delta E)=Q(-\Delta E) \exp (-\beta \Delta E)$.
} 


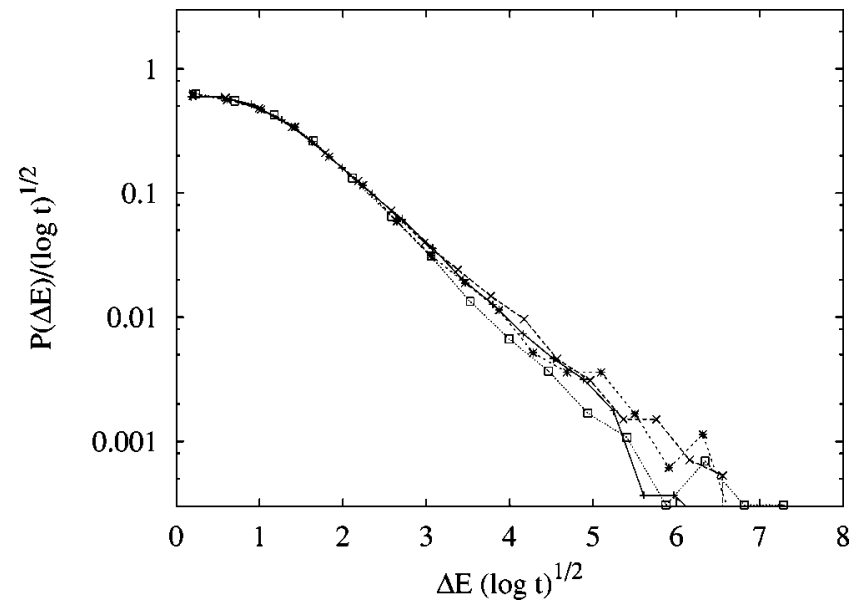

FIG. 11. Scaling plot for $P(\Delta E) / \sqrt{\ln (t)}$ versus $\Delta E \sqrt{\ln (t)}$ for different times $t=10^{2}, 10^{3}, 10^{4}, 10^{5}$.

that the range where $P(\Delta E)$ is finite corresponds to the region where $\epsilon \sim \epsilon^{*}$. In Fig. 11 this corresponds to $\epsilon^{*}$ $\simeq 6 / \sqrt{\ln (t)}$ in agreement with what was observed in Figs. 8 and 9 .

The scaling works pretty well showing how this method could be used to guess the time evolution of the energy threshold $\epsilon^{*}$ in general glassy models in those cases where there exists an energy threshold.

\section{CONCLUSIONS}

In this paper we have considered a solvable glass model, the DBG. The new ingredient of this model is that each box has a positive random energy associated with it, which is obtained from a distribution $g(\epsilon)$. Again, similarly to its predecessor $(\mathrm{BG})$, the model displays slow relaxation due to the presence of entropic barriers. Actually, it turns out that the relaxation at $T=0$ of the number of empty boxes and all other occupation probabilities $P_{k}$ is exactly the same as the original BG model, and, in particular, independent of the disorder distribution $g(\epsilon)$. In general, the relaxation of other quantities such as the energy and other disorder dependent observables, displays an asymptotic relaxation that depends on the statistical properties of $g(\epsilon)$ in the limit $\epsilon \rightarrow 0$. In the asymptotic long-time regime, relaxation takes place by diffusing particles among boxes with the smallest values of $\epsilon$. Therefore the asymptotic decay of the energy, as well as that of other observables, only depends on the exponent $\alpha$ that describes the limiting behavior $g(\boldsymbol{\epsilon}) \rightarrow \epsilon^{\alpha}$. The original BG model is recovered as a special case in the limit $\alpha \rightarrow \infty$.

We have written the integral equations for the densities $g_{k}(\epsilon)$. These equations form a hierarchy of dynamical equations which can be closed by introducing a suitable generating function. We focused on the solution of this hierarchy in the particular case of zero temperature. In this case the analytical solution of these equations proceeds in two steps. First, the equations for the occupancies $P_{k}$ are exactly the same as in the original BG model and they can be solved by using known analytical methods. Second, this information is used to guess an adiabatic solution for the $g_{k}(\epsilon)$ in terms of a time-dependent energy threshold $\epsilon^{*}$.

All densities are out of equilibrium but admit a scaling solution of the type $\hat{g}_{k}\left(\epsilon / \epsilon^{*}\right)$, with the condition that, for $\epsilon$ $>\epsilon^{*}, \hat{g}_{k}$ decays to zero fast enough to guarantee that $\int_{0}^{\infty} d x \hat{g}_{k}(x)=1$. That means that for energies above the threshold the modes are almost completely thermalized.

This ansatz solution yields two types of leading behaviors: on one hand, they yield the asymptotic long-time behavior of $\epsilon^{*}, z^{*}$ at zero temperature, while on the other hand they produce the low-temperature behavior of the values of $\epsilon^{*}, z^{*}$ in the stationary equilibrium limit $t \rightarrow \infty$. The adiabatic approximation is nothing else but stating the validity of the complementary description of these two very different regimes. On one hand is the equilibrium regime, where first the limit $t \rightarrow \infty$ is taken and later $T \rightarrow 0$, while on the other hand is the far from equilibrium regime, where the limit $T \rightarrow 0$ is taken first and later $t \rightarrow \infty$. The commutation of these two limits allows for the interchange between different variables such as energy, temperature, and times when expressed in terms of their asymptotic leading behavior. Knowing the leading behavior of the quantities $z^{*}$ and $\epsilon^{*}$, dimensional reasoning as presented in Appendix B yields the leading behavior of $\beta_{\text {eff }}$, which turns out to be proportional to $z^{*} / \epsilon^{*}$. The DBG model offers a scenario where there are two energy sectors separated by the energy scale $\epsilon^{*}$, which have very different physical properties. These two sectors manifest in the behavior of observables such as the probability densities $g_{k}(\epsilon)$ where the time dependent threshold $\epsilon^{*}$ separates the equilibrated modes $\left(\epsilon>\epsilon^{*}\right)$ from the modes that stay off equilibrium $\left(\epsilon<\epsilon^{*}\right)$. In the off-equilibrium regime, entropic barriers are typically higher than the time-dependent barrier at the threshold level $\epsilon^{*}$. For $\epsilon>\epsilon^{*}$ barriers are lower and equilibrium is achieved faster.

\section{DISCUSSION}

How is the existence of this energy scale of interest? One of the most interesting properties of the present model is that it introduces, in a very simple way, the concept of a threshold energy scale. To our knowledge, such concept has never been discussed in any one among the plethora of mean-field glassy models studied during recent years. In those cases, one studies the relaxation of global quantities that get contribution of all possible energy scales involved into the problem. In principle, nothing is wrong with that since the macroscopic observables are those quantities that are always measured in the laboratory. The problem arises when tackling issues related to the violation of the fluctuation-dissipation theorem and concepts such as the effective temperature and partial equilibration. Usually an effective temperature is defined in terms of the measured dissipation (response) and fluctuations (correlations) in the aging regime. This effective temperature is supposed to quantify the amount of energy transfer when the system is put in contact with a thermal bath and behaves in several aspects as a real temperature $[14,16,34]$. There is a fundamental problem with this definition, which is the following. Suppose one takes a piece of silica well below $T_{g}$ 
(for instance, at room temperature). The piece of silica is not in equilibrium (actually, it is always relaxing even if the relaxation rate is extremely small and unobservable at room temperature) so one would be tempted to claim that its effective temperature (that describing the equipartition among the set of nonthermalized degrees of freedom) is around $T_{g}$ well above the room temperature. Obviously, if we touch a piece of silica, then the hand plays the role of a thermal bath at the room temperature. Therefore, why we do not feel the effective temperature that may be hundreds of degrees above the room temperature? Note that the energy content of the glass is still very large. Indeed, if the glass suddenly crystallized it would liberate all its latent heat [33]. There are two possible explanations of discrepancy. The first one was analyzed in the context of the oscillator model and assumes that the thermal conductivity is so small that the heat transfer is negligible over such a short time scale [34]. The other explanation is that, when touching the glass, we are not touching the slow collective degrees of freedom that still contain a lot of energy but the thermalized degrees of freedom. These two explanations are not totally exclusive. Assuming the existence of an energy threshold $\epsilon^{*}$ such that, above $\epsilon^{*}$, all collective modes are thermalized at the bath temperature and below that threshold they are off equilibrium, this offers an explanation about why when touching a piece of glass we feel it at the room temperature. Our hand only couples to the higher energy degrees of freedom (phonons) and not to the (much hotter) collective excitations. Yet, we cannot exclude that even if we coupled the bath to the hottest collective degrees of freedom then the conductivity would be extremely small and no heat transfer would be measured. From a different point of view, the two different explanations for the small amount of heat transfer established between a bath and the "hot" glassy system reduce to a single one: the existence of a threshold scale $\epsilon^{*}$ is a consequence of the highly different orders of magnitude for the conductivities in the two energy sectors. Future studies of other glassy models will better clarify this issue [35].

Finally, we have proposed a method to determine the threshold energy scale $\epsilon^{*}$ by computing the general probability distribution $Q(\Delta E)$. Preliminary investigations in other glassy models show that this distribution provides a general way to determine the threshold scale $\epsilon^{*}$. Moreover, it gives interesting information about fluctuations in the aging state although future work is still needed to understand better its full implications in our understanding of the aging regime.

Further investigations in this model will address other issues such as the measurement of effective temperatures. For instance, it would be interesting to understand how the effective temperature, defined as the temperature of the thermal bath, which does not produce a net thermal current when put in contact with the system, depends on the energy sector $\epsilon$ probed by the bath. By coupling the bath with a selected set of modes of energy $\epsilon$ we can understand whether there is a single effective temperature $T_{\text {eff }}$ for all modes or rather, there is an $\epsilon$ dependent temperature. Note that the local equilibrium in this model is only valid in the energy sector $\epsilon>\epsilon^{*}$ and it could well be that there does not exist a well defined
TABLE I. Processes involved in the dynamics of the probability density $g_{0}(\epsilon)$ of empty boxes at energy $\epsilon$.

\begin{tabular}{ccc}
\hline \hline \multicolumn{2}{c}{ Occupation } & Contribution to $\Delta g_{0}(\epsilon)$ \\
\hline$n_{d}=1$ & $n_{a}=0$ & $\left.\delta_{n_{d}, 1} \delta_{n_{a}, 0} \delta\left(\epsilon-\epsilon_{d}\right)-\delta\left(\epsilon-\epsilon_{a}\right)\right]$ \\
& & $\times\left[1+\left(e^{-\beta\left(\epsilon_{a}-\epsilon_{d}\right)}-1\right) \theta\left(\epsilon_{a}-\epsilon_{d}\right)\right]$ \\
& $n_{a}>0$ & $\delta_{n_{d}, 1}\left(1-\delta_{n_{a}, 0}\right) \delta\left(\epsilon-\epsilon_{d}\right)$ \\
$n_{d}>1$ & $n_{a}=0$ & $-\left(1-\delta_{n_{d}, 1}\right) \delta_{n_{a}, 0} e^{-\beta \epsilon_{a}} \delta\left(\epsilon-\epsilon_{a}\right)$ \\
\hline
\end{tabular}

effective temperature in the other sector $\epsilon<\epsilon^{*}$. Nevertheless, the most natural possibility is that the thermal fluctuations in the off-equilibrium sector $\epsilon<\epsilon^{*}$ are determined by the effective temperature [see Eq. (32)] that determines the relaxation rate of the slow collective mode $P_{0}$. Future studies should enlighten this and other related questions.

\section{ACKNOWLEDGMENTS}

We acknowledge A. Garriga and Th. M. Nieuwenhuizen for a careful reading of the manuscript and suggestions. L.L. is supported by FOM, The Netherlands. He acknowledges financial support from LPTHE where part of this work was done. F.R. is supported by the Spanish Ministerio de Ciencia y Tecnología, project PB97-0971. F.R. acknowledges support from the French-Spanish Collaboration (Picasso program and Acciones Integradas HF2000-0097).

\section{APPENDIX A: OCCUPATION PROBABILITY DENSITY EQUATIONS}

In this appendix we derive the equations of motion for the occupation probability densities for box energy between $\epsilon$ and $\epsilon+d \epsilon$. First we start from the densities of having zero particle in a box of energy $\epsilon$.

In Table I we list the processes contributing to the evolution of the occupation probability density of boxes containing zero particles. In the left column, we show the processes involved in terms of occupation numbers of the departure box and of the arrival box. In the right column, we write the correspondent contribution of a given process to the variation of the occupation density, $\Delta g_{0}(\epsilon)$.

The particle for which a jump is proposed is chosen in box $d$ with probability $n_{d} / N$. The arrival box is chosen with uniform probability $1 / N$.

The total difference per particle in the probability density of empty boxes of energy $\epsilon$ is then

$$
\begin{aligned}
\Delta g_{0}(\epsilon)= & \frac{1}{N} \sum_{p=0}^{N} \sum_{a=0}^{N} \frac{n_{p}}{N} \frac{1}{N}\left\{\delta_{n_{d}, 1} \delta_{n_{a}, 0}\left[\delta\left(\epsilon-\epsilon_{d}\right)-\delta\left(\epsilon-\epsilon_{a}\right)\right]\right. \\
& \times\left[1+\left(e^{-\beta\left(\epsilon_{a}-\epsilon_{d}\right)}-1\right) \theta\left(\epsilon_{a}-\epsilon_{d}\right)\right] \\
& +\delta_{n_{d}, 1}\left(1-\delta_{n_{a}, 0}\right) \delta\left(\epsilon-\epsilon_{d}\right)-\left(1-\delta_{n_{d}, 1}\right) \\
& \left.\times \delta_{n_{a}, 0} e^{-\beta \epsilon_{a}} \delta\left(\epsilon-\epsilon_{a}\right)\right\} .
\end{aligned}
$$

Using Eqs. (3)-(6) and the following identities: 
TABLE II. Processes contributing to the dynamics of $g_{1}(\epsilon)$.

\begin{tabular}{ccc}
\hline \hline \multicolumn{2}{c}{ Occupation } & Contribution to $\Delta g_{1}(\epsilon)$ \\
\hline$n_{d}=1$ & $n_{a}=0$ & $\delta_{n_{d}, 1} \delta_{n_{a}, 0}\left[\delta\left(\epsilon-\epsilon_{d}\right)-\delta\left(\epsilon-\epsilon_{a}\right)\right]$ \\
& & $\times\left[1+\left(e-\beta\left(\epsilon_{a}-\epsilon_{d}\right)-1\right) \theta\left(\epsilon_{a}-\epsilon_{d}\right)\right]$ \\
& $n_{a}=1$ & $-\delta_{n_{d}, 1} \delta_{n_{a}, 1}\left[\delta\left(\epsilon-\epsilon_{d}\right)+\delta\left(\epsilon-\epsilon_{a}\right)\right]$ \\
& $n_{a}>1$ & $-\delta_{n_{d}, 1}\left(1-\delta_{n_{a}, 1}-\delta_{n_{a}, 0}\right) \delta\left(\epsilon-\epsilon_{d}\right)$ \\
$n_{d}=2$ & $n_{a}=0$ & $\delta_{n_{d}, 2} \delta_{n_{a}, 0}\left[\delta\left(\epsilon-\epsilon_{d}\right)+\delta\left(\epsilon-\epsilon_{a}\right)\right] e^{-\beta \epsilon_{a}}$ \\
& $n_{a}=1$ & $\delta_{n_{d}, 2} \delta_{n_{a}, 1}\left[\delta\left(\epsilon-\epsilon_{d}\right)-\delta\left(\epsilon-\epsilon_{a}\right)\right]$ \\
& $n_{a}>1$ & $\delta_{n_{d}, 2}\left(1-\delta_{n_{a}, 1}-\delta_{n_{a}, 0}\right) \delta\left(\epsilon-\epsilon_{d}\right)$ \\
$n_{d}>2$ & $n_{a}=0$ & $\left(1-\delta_{n_{d}, 2}-\delta_{n_{d}, 1}\right) \delta_{n_{a}, 0} \delta\left(\epsilon-\epsilon_{a}\right) e^{-\beta \epsilon_{a}}$ \\
& $n_{a}=1$ & $-\left(1-\delta_{n_{d}, 2}-\delta_{n_{d}, 1}\right) \delta_{n_{a}, 1} \delta\left(\epsilon-\epsilon_{a}\right)$ \\
\hline \hline
\end{tabular}

$$
\begin{gathered}
\frac{1}{N} \sum_{a=0}^{N} \delta_{n_{a}, 0} \theta\left(\epsilon_{a}-\epsilon\right)\left[e^{-\beta\left(\epsilon_{a}-\epsilon\right)}-1\right] \\
\quad=\int_{\epsilon}^{\infty} d \epsilon^{\prime} g_{0}\left(\epsilon^{\prime}\right)\left[e^{-\beta\left(\epsilon^{\prime}-\epsilon\right)}-1\right],
\end{gathered}
$$$$
\frac{1}{N} \sum_{d=0}^{N} n_{d} \delta_{n_{d}, 1} \theta\left(\epsilon-\epsilon_{d}\right)\left[e^{-\beta\left(\epsilon-\epsilon_{d}\right)}-1\right]
$$$$
=\int_{0}^{\epsilon} d \epsilon^{\prime} g_{1}\left(\epsilon^{\prime}\right)\left[e^{-\beta\left(\epsilon-\epsilon^{\prime}\right)}-1\right],
$$$$
\frac{1}{N} \sum_{a=0}^{N} \delta_{n_{a}, 0} \delta\left(\epsilon-\epsilon_{a}\right) e^{-\beta \epsilon_{a}}=g_{0}(\epsilon) e^{-\beta \epsilon}
$$

we get the equation of motion for $g_{0}(\epsilon)$ [namely, Eq. (24)],

$$
\begin{aligned}
\frac{\partial g_{0}(\epsilon)}{\partial t}= & \lim _{N \rightarrow \infty} \frac{\Delta g_{0}(\epsilon)}{1 / N} \\
= & g_{1}(\epsilon)\left[1+\int_{\epsilon}^{\infty} d \epsilon^{\prime} g_{0}\left(\epsilon^{\prime}\right)\left(e^{-\beta\left(\epsilon^{\prime}-\epsilon\right)}-1\right)\right] \\
& -g_{0}(\epsilon)\left[e^{-\beta \epsilon}+P_{1}\left(1-e^{-\beta \epsilon}\right)\right. \\
& \left.+\int_{0}^{\epsilon} d \epsilon^{\prime} g_{1}\left(\epsilon^{\prime}\right)\left(e^{-\beta\left(\epsilon-\epsilon^{\prime}\right)}-1\right)\right] .
\end{aligned}
$$

We then consider the evolution of the probability density for boxes containing one particle. In Table II we list the processes contributing to the evolution of such occupation probability density.

Departure boxes are chosen with probability $n_{d} / N$. Arrival boxes are chosen with uniform probability $1 / N$.

Using again Eqs. (3)-(6) and Eqs. (A3),(A5) we are able to derive the equation of motion for the probability density of boxes with one particles and energy equal to $\epsilon$,
TABLE III. List of the processes involved in the dynamics of the probability density $g_{k}(\epsilon)$, for $k>1$.

\begin{tabular}{ccc}
\hline \hline \multicolumn{2}{c}{ Occupation } & Contribution to $\Delta g_{k}(\epsilon)$ \\
\hline$n_{d}=h<k$ & $n_{a}=k-1$ & $\delta_{n_{d}, h} \delta_{n_{a}, k-1} \delta\left(\epsilon-\epsilon_{a}\right)$ \\
& $n_{a}=k$ & $-\delta_{n_{d}, h} \delta_{n_{a}, k} \delta\left(\epsilon-\epsilon_{a}\right)$ \\
$n_{d}=k$ & $n_{a}=0$ & $-\delta_{n_{d}, k} \delta_{n_{a}, 0} \delta\left(\epsilon-\epsilon_{a}\right) e^{-\beta \epsilon_{a}}$ \\
& $0<n_{a}=h<k-1$ & $-\delta_{n_{d}, k} \delta_{n_{a}, h} \delta\left(\epsilon-\epsilon_{a}\right)$ \\
& $n_{a}=k-1$ & $-\delta_{n_{d}, k} \delta_{n_{a}, k-1}\left[\delta\left(\epsilon-\epsilon_{d}\right)-\delta\left(\epsilon-\epsilon_{a}\right)\right]$ \\
& $n_{a}=k$ & $-\delta_{n_{d}, k} \delta_{n_{a}, k}\left[\delta\left(\epsilon-\epsilon_{d}\right)+\delta\left(\epsilon-\epsilon_{a}\right)\right]$ \\
& $n_{a}>k$ & $-\delta_{n_{d}, k}\left(1-\sum_{h=0}^{k} \delta_{n_{a}, h}\right) \delta\left(\epsilon-\epsilon_{d}\right)$ \\
$n_{d}=k+1$ & $n_{a}=0$ & $-\delta_{n_{d}, k+1} \delta_{n_{a}, 0} \delta\left(\epsilon-\epsilon_{d}\right) e^{-\beta \epsilon_{a}}$ \\
& $0<n_{a}<k-1$ & $\delta_{n_{d}, k+1} \delta_{n_{a}, h} \delta\left(\epsilon-\epsilon_{d}\right)$ \\
& $n_{a}=k-1$ & $\delta_{n_{d}, k+1} \delta_{n_{a}, k-1}\left[\delta\left(\epsilon-\epsilon_{d}\right)+\delta\left(\epsilon-\epsilon_{a}\right)\right]$ \\
& $n_{a}=k$ & $\delta_{n_{d}, k+1} \delta_{n_{a}, k}\left[\delta\left(\epsilon-\epsilon_{d}\right)-\delta\left(\epsilon-\epsilon_{a}\right)\right]$ \\
& $n_{a}>k$ & $\delta_{n_{d}, k+1}\left(1-\sum_{h=0}^{k} \delta_{n_{a}, h}\right) \delta\left(\epsilon-\epsilon_{d}\right)$ \\
$n_{d}>k$ & $n_{a}=k-1$ & $\left(1-\sum_{h=1}^{k+1} \delta_{n_{d}, h}\right) \delta_{n_{a}, k-1} \delta\left(\epsilon-\epsilon_{a}\right)$ \\
& $n_{a}=k$ & $-\left(1-\sum_{h=1}^{k+1} \delta_{n_{d}, h}\right) \delta_{n_{a}, k y} \delta\left(\epsilon-\epsilon_{a}\right)$ \\
\hline \hline
\end{tabular}

$$
\begin{aligned}
\frac{\partial g_{1}(\epsilon)}{\partial t}= & 2 g_{2}(\epsilon)\left(1+\int_{0}^{\infty} d \epsilon g_{0}(\epsilon) e^{-\beta \epsilon}-P_{0}\right) \\
& -g_{1}(\epsilon)\left[2+\int_{\epsilon}^{\infty} d \epsilon^{\prime} g_{0}\left(\epsilon^{\prime}\right)\left(e^{-\beta\left(\epsilon^{\prime}-\epsilon\right)}-1\right)\right] \\
& +g_{0}(\epsilon)\left[e^{-\beta \epsilon}+P_{1}\left(1-e^{-\beta \epsilon}\right)\right. \\
& \left.+\int_{0}^{\epsilon} d \epsilon^{\prime} g_{1}\left(\epsilon^{\prime}\right)\left(e^{-\beta\left(\epsilon-\epsilon^{\prime}\right)}-1\right)\right] .
\end{aligned}
$$

For densities of boxes with $k>1$ particle the scheme of the contributions is presented in Table III. (26)

Combining all the contributions we obtain for $g_{k}(\epsilon) \mathrm{Eq}$.

$$
\begin{aligned}
\frac{\partial g_{k}(\boldsymbol{\epsilon})}{\partial t}= & (k+1) g_{k+1}(\boldsymbol{\epsilon})\left(1+\int_{0}^{\infty} d \epsilon g_{0}(\boldsymbol{\epsilon}) e^{-\beta \epsilon}-P_{0}\right) \\
& -g_{k}(\boldsymbol{\epsilon})\left[1+k+k\left(\int_{0}^{\infty} d \epsilon g_{0}(\boldsymbol{\epsilon}) e^{-\beta \epsilon}-P_{0}\right)\right] \\
& +g_{k-1}(\boldsymbol{\epsilon}) .
\end{aligned}
$$

\section{APPENDIX B: ANSATZ FOR THE ADIABATIC APPROXIMATION}

In this appendix we show that the ansatz solution (40) is asymptotically a solution of Eqs. (33) $-(35)$ at $T=0$ yielding the leading behavior $\epsilon^{*}(41)$. We start by rewriting Eq. (40) in the following way:

$$
\Delta g_{k}(\epsilon)=\frac{\Delta P_{k}}{\epsilon} h_{k}\left(\frac{\epsilon}{\epsilon^{*}}\right),
$$

where $\Delta P_{k} \equiv P_{k}-\delta_{k, 0}, \Delta g_{k}(\epsilon) \equiv g_{k}(\epsilon)-\delta_{k, 0} g(\epsilon), h_{k}(x)$ $=x \hat{g}_{k}(x)$, and $\int_{0}^{\infty} d x \hat{g}_{k}(x)=\int_{0}^{\infty} d x h_{k}(x) / x=1$. Here we will 
perform the analysis for the case $k=0$. The equations for $k$ $>0$ can be obtained in a similar fashion. Substituting this expression into Eq. (33) we get

$$
\begin{aligned}
\frac{\partial g_{0}(\epsilon)}{\partial t}= & \frac{\partial \Delta P_{0}}{\partial t} \frac{1}{\epsilon} h_{0}\left(\frac{\epsilon}{\epsilon^{*}}\right)-\frac{\Delta P_{0}}{\left(\epsilon^{*}\right)^{2}} h_{0}^{\prime}\left(\frac{\epsilon}{\epsilon^{*}}\right) \frac{d \epsilon^{*}(t)}{d t} \\
= & -\frac{\Delta P_{1}}{\epsilon} h_{1}\left(\frac{\epsilon}{\epsilon^{*}}\right)\left[\int_{0}^{\epsilon} d \epsilon^{\prime} g\left(\epsilon^{\prime}\right)\right. \\
& \left.-\Delta P_{0} \int_{\epsilon}^{\infty} d \epsilon^{\prime} \frac{1}{\epsilon^{\prime}} h_{0}\left(\frac{\epsilon}{\epsilon^{*}}\right)\right] \\
& +\Delta P_{1}\left[g(\epsilon)+\frac{\Delta P_{0}}{\epsilon} h_{0}\left(\frac{\epsilon}{\epsilon^{*}}\right)\right. \\
& \left.\times \int_{\epsilon}^{\infty} d \epsilon^{\prime} \frac{1}{\epsilon^{\prime}} h_{1}\left(\frac{\epsilon}{\epsilon^{*}}\right)\right],
\end{aligned}
$$

where $h_{0}^{\prime}(x)$ stands for the first derivative of $h_{0}(x)$. Note that the scaling function $h_{0}$ does not depend on time, hence there is no term $\partial h_{0} / \partial t$ in that expression. Now introduce Eq. (29) in the first term on the left-hand side of Eq. (B2) and multiply the whole equation by $\epsilon / \Delta P_{0}$ to obtain

$$
\begin{aligned}
\Delta P_{1} & h_{0}(x)+x h_{0}^{\prime}(x) \frac{\partial \ln \left(\epsilon^{*}\right)}{\partial t} \\
= & \Delta P_{1} h_{1}(x)\left[\frac{1}{\Delta P_{0}} \int_{0}^{\epsilon} d \epsilon^{\prime} g\left(\epsilon^{\prime}\right)-\int_{x}^{\infty} d x^{\prime} \hat{g}_{0}(x)\right] \\
& -\left[\frac{\epsilon g(\epsilon)}{\Delta P_{0}}+h_{0}(x)\right] \Delta P_{1} \int_{x}^{\infty} d x^{\prime} \hat{g}_{1}(x),
\end{aligned}
$$

where $\hat{g}_{k}(x)=\left[h_{k}(x) / x\right]$. From this equation we can guess the scaling behavior of all quantities in the asymptotic largetime limit $\epsilon^{*} \rightarrow 0$. In the sector $\epsilon \leqslant \epsilon^{*}$ we use $g(\epsilon) \sim \epsilon^{\alpha}$ obtaining $\int_{0}^{\epsilon} d \epsilon^{\prime} g\left(\epsilon^{\prime}\right) \sim \epsilon^{\alpha+1}$. Assuming all terms of the same order, we get for $\epsilon \sim \epsilon^{*}$,

$$
\begin{gathered}
\Delta P_{0} \sim\left(\epsilon^{*}\right)^{\alpha+1}, \\
\Delta P_{1} \sim-\frac{\partial \ln \left(\epsilon^{*}\right)}{\partial t} .
\end{gathered}
$$

Using the standard adiabatic results (31), $P_{0}=1$ $-1 / z^{*}, P_{1}=1 /\left[\exp \left(z^{*}\right)\right]$ we obtain the results $(41)$ and $(42)$. Note that the set of equations for $h_{k}$ are still impossible to solve. Only in certain regimes such as $\epsilon \ll \epsilon^{*}$ it may be possible to obtain results. There is a set of equations which couples the different $h_{k}$. But this set of equations is time independent and should yield all the scaling functions $\hat{g}_{k}(x)$ once appropriate treatment is done of the amplitude constant, which fixes the leading behavior of $\epsilon^{*}$.

We also consider, as an example, the case in which the probability distribution of the quenched disorder becomes exponentially high at high values of $\epsilon$ and zero for low values, namely, we choose

$$
g(\epsilon)=\exp \left(-\frac{A}{\epsilon}\right) .
$$

For this choice $\int_{0}^{\epsilon} d \epsilon^{\prime} g\left(\epsilon^{\prime}\right) \sim-\epsilon \exp (-A / \epsilon)-A \Gamma(0, A / \epsilon)$, where the generalized Euler function $\Gamma(0, x)$ goes to zero as $x \rightarrow \infty$. In order to estimate $\epsilon^{*}$ from Eq. (B3) we notice now that for $P_{1}$ Eq. (B5) is still valid, while for $\Delta P_{0}$ we obtain

$$
\Delta P_{0} \sim-\epsilon^{*} \exp \left(-\frac{A}{\epsilon^{*}}\right)
$$

eventually yielding

$$
\epsilon^{*}(t) \sim \frac{A}{\ln [\ln (t)]} .
$$

\section{APPENDIX C: APPROACH TO EQUILIBRIUM OF THE OCCUPATION DENSITIES $g_{k}(\epsilon)$}

We present the equations of motions for the occupation densities in the asymptotic regime. The values of the densities are expanded to first order around their equilibrium values: $g_{k}=g_{k}^{\mathrm{eq}}+\delta g_{k}$,

$$
\begin{aligned}
\frac{\partial \delta g_{0}(\epsilon)}{\partial t}= & \delta g_{1}(\epsilon)\left\{1+\int_{\epsilon}^{\infty} d \epsilon^{\prime} g_{0}^{\mathrm{eq}}\left(\epsilon^{\prime}\right)\left[e^{-\beta\left(\epsilon^{\prime}-\epsilon\right)}-1\right]\right\} \\
& -\delta g_{0}(\epsilon)\left[e^{-\beta \epsilon}+P_{1}^{\mathrm{eq}}\left(1-e^{-\beta \epsilon}\right)\right. \\
& \left.+z \int_{0}^{\epsilon} d \epsilon^{\prime} g_{0}^{\mathrm{eq}}\left(\epsilon^{\prime}\right)\left(e^{-\beta \epsilon}-e^{\left.-\beta \epsilon^{\prime}\right)}\right)\right]+g_{0}^{\mathrm{eq}}(\epsilon) \\
& \times\left\{z \int_{\epsilon}^{\infty} d \epsilon^{\prime} \delta g_{0}\left(\epsilon^{\prime}\right)\left(e^{-\beta \epsilon^{\prime}}-e^{-\beta \epsilon}\right)\right. \\
& -\left(1-e^{-\beta \epsilon}\right) \int_{0}^{\infty} d \epsilon^{\prime} \delta g_{1}\left(\epsilon^{\prime}\right) \\
& \left.-\int_{0}^{\epsilon} d \epsilon^{\prime} \delta g_{1}\left(\epsilon^{\prime}\right)\left[e^{-\beta\left(\epsilon-\epsilon^{\prime}\right)}-1\right]\right\}, \quad k=0,
\end{aligned}
$$


TABLE IV. Contributions to the probability distribution $Q(\Delta E)$ of proposed energy updates.

\begin{tabular}{cccc}
\hline \hline \multicolumn{2}{c}{ Occupation } & Contribution to $E^{\prime}-E$ & Probability \\
\hline$n_{d}=1$ & $n_{a}=0$ & $-\epsilon_{d}+\epsilon_{a}$ & $g_{1}\left(\epsilon_{d}\right) g_{0}\left(\epsilon_{a}\right)$ \\
$n_{d}>1$ & $n_{a}>0$ & $-\epsilon_{d}$ & $g_{1}\left(\epsilon_{d}\right)\left[g\left(\epsilon_{a}\right)-g_{0}\left(\epsilon_{a}\right)\right]$ \\
& $n_{a}=0$ & $\epsilon_{a}$ & $g_{0}\left(\epsilon_{a}\right) \frac{1}{N} \Sigma_{p} n_{p}\left[g\left(\epsilon_{d}\right)-g_{1}\left(\epsilon_{d}\right)\right]$ \\
& $n_{a}>0$ & 0 & {$\left[g\left(\epsilon_{a}\right)-g_{0}\left(\epsilon_{a}\right)\right] \frac{1}{N} \Sigma_{p} n_{p}\left[g\left(\epsilon_{d}\right)-g_{1}\left(\epsilon_{d}\right)\right]$} \\
\hline \hline
\end{tabular}

$$
\begin{aligned}
\frac{\partial \delta g_{1}(\epsilon)}{\partial t}= & \frac{2}{z} \delta g_{2}(\epsilon)-\delta g_{1}(\epsilon)\left[2+\int_{\epsilon}^{\infty} d \epsilon^{\prime} g_{0}\left(\epsilon^{\prime}\right)\left[e^{-\beta\left(\epsilon^{\prime}-\epsilon\right)}\right.\right. \\
& -1]]+\delta g_{0}(\epsilon)\left[e^{-\beta \epsilon}+P_{1}^{\mathrm{eq}}\left(1-e^{-\beta \epsilon}\right)\right. \\
& \left.+z \int_{0}^{\epsilon} d \epsilon^{\prime} g_{0}\left(\epsilon^{\prime}\right)\left(e^{-\beta \epsilon}-e^{-\beta \epsilon}\right)\right]-g_{0}^{\mathrm{eq}}(\epsilon) \\
& \times\left\{z^{2} e^{-\beta \epsilon} \int_{0}^{\infty} d \epsilon^{\prime} \delta g_{0}\left(\epsilon^{\prime}\right)\left(1-e^{-\beta \epsilon^{\prime}}\right)\right. \\
& +z \int_{\epsilon}^{\infty} d \epsilon^{\prime} \delta g_{0}\left(\epsilon^{\prime}\right)\left(e^{-\beta \epsilon^{\prime}}-e^{-\beta \epsilon}\right) \\
& -\left(1-e^{-\beta \epsilon}\right) \int_{0}^{\infty} d \epsilon^{\prime} \delta g_{1}\left(\epsilon^{\prime}\right)-\int_{0}^{\epsilon} d \epsilon^{\prime} \delta g_{1}\left(\epsilon^{\prime}\right) \\
& \left.\times\left[e^{-\beta\left(\epsilon-\epsilon^{\prime}\right)}-1\right]\right\}, \quad k=1,
\end{aligned}
$$$$
\frac{\partial \delta g_{k}(\epsilon)}{\partial t}=\delta g_{k+1}(\epsilon) \frac{k+1}{z}-\delta g_{k}(\epsilon)\left(1+\frac{k}{z}\right)+\delta g_{k-1}(\epsilon)
$$$$
-g_{0}^{\mathrm{eq}}(\epsilon) \frac{z^{k+1}}{k !} e^{-\beta \epsilon}\left(1-\frac{k}{z}\right) \int_{0}^{\infty} d \epsilon^{\prime} \delta g_{0}\left(\epsilon^{\prime}\right)
$$$$
\times\left(1-e^{-\beta \epsilon}\right), \quad k>1 .
$$

In the above equations $\beta$ is the inverse thermal bath temperature and $z$ is the equilibrium fugacity at that temperature.

As $T$ goes to zero $[\beta \rightarrow \infty, z(\beta) \rightarrow \infty]$ the equations for the first order perturbation to equilibrium can be closed,

$$
\begin{aligned}
\frac{\partial \delta g_{0}(\epsilon)}{\partial t}= & \delta g_{1}(\epsilon)-\int_{\epsilon}^{\infty} d \epsilon^{\prime}\left[\delta g_{1}(\epsilon) g\left(\epsilon^{\prime}\right)\right. \\
& \left.+g(\epsilon) \delta g_{1}\left(\epsilon^{\prime}\right)\right], \quad k=0, \\
\frac{\partial \delta g_{1}(\epsilon)}{\partial t}= & -2 \delta g_{1}(\epsilon)+\int_{\epsilon}^{\infty} d \epsilon^{\prime}\left[\delta g_{1}(\epsilon) g\left(\epsilon^{\prime}\right)\right. \\
& \left.+g(\epsilon) \delta g_{1}\left(\epsilon^{\prime}\right)\right], \quad k=1, \\
\frac{\partial \delta g_{k}(\epsilon)}{\partial t}= & -\delta g_{k}(\epsilon)+\delta g_{k-1}(\epsilon), \quad k>1 .
\end{aligned}
$$

In order to estimate the relaxation characteristic time to equilibrium at low temperature we can expand Eqs. (C1). First we introduce the asymptotic threshold energy $\epsilon^{*}(T)$ as the energy discriminating between the thermalized and the nonthermalized collective modes at temperature $T$. If we define it through the relation $\epsilon^{*}(T)=T z(T)$ and use the relation (22) obtained by doing a low- $T$ expansion then we get

$$
\epsilon^{*}(T)=z_{0} T^{1 /(2+\alpha)},
$$

where $z_{0}$ is the coefficient of the leading term of $z(T)$ at low $T$ [see Eq. (22), $z(T)=z_{0} T^{(1+\alpha) /(2+\alpha)}$ ].

Then we expand Eqs. (C1), take $\epsilon \simeq \epsilon^{*}$, and introduce the following adiabatic ansatz:

$$
\delta g_{k}(\epsilon) \equiv g_{k}(\epsilon)-g_{k}^{\mathrm{eq}}(\epsilon)=\frac{\Delta P_{k}(T, t)}{\epsilon^{*}(T)} \hat{g}_{k}\left(\frac{\epsilon}{\epsilon^{*}(T)}\right) .
$$

Note that this solution is equivalent to the ansatz Eq. (40) introduced for the asymptotic dynamics at zero temperature but with a static $\epsilon^{*}(T)$ now replacing the dynamical threshold. Now consider Eq. (C1) for $\delta g_{0}(\epsilon)$. Because $\delta P_{k}$ $=\int d \epsilon \delta g_{k}(\epsilon)$ it can be shown that the slowest mode corresponds to $k=0$, i.e., $\delta g_{0}(\epsilon) \gg \delta g_{k}(\epsilon)$ for $k>0$. Therefore the second term in the right-hand side of Eq. (C1) dominates the first and the second terms. Introducing Eq. (C8) into Eq. (C1) we get that the relaxation time behaves like

$$
\tau_{\text {eq }}\left(\epsilon^{*}\right) \propto \frac{e^{\beta \epsilon^{*}}}{\beta \epsilon^{*}} .
$$

For $\epsilon \gg \epsilon^{*}$ the relaxation time is much smaller, since those are the modes with lower energy barriers.

\section{APPENDIX D: PROBABILITY DISTRIBUTION OF PROPOSED ENERGY UPDATES}

In this appendix the probability distribution of proposed energy updates is built. In Table IV we summarize all the processes contributing to it, together with their probabilities.

The probability distribution $Q(\Delta E)$ of proposed energy updates is the average of all possible changes, each computed with its probability,

$$
Q(\Delta E) \equiv \overline{\delta\left(E^{\prime}-E-\Delta E\right)},
$$

where $\Delta E$ is the proposed update, $E$ is the energy of the system before the updating, and $E^{\prime}$ the energy afterwards. This means 


$$
\begin{aligned}
Q(\Delta E)= & \int_{0}^{\infty} d \boldsymbol{\epsilon} \int_{0}^{\infty} d \epsilon^{\prime} g_{1}(\boldsymbol{\epsilon}) g_{0}\left(\boldsymbol{\epsilon}^{\prime}\right) \delta\left(\Delta+\boldsymbol{\epsilon}-\boldsymbol{\epsilon}^{\prime}\right)+\int_{0}^{\infty} d \epsilon \int_{0}^{\infty} d \boldsymbol{\epsilon}^{\prime} g_{1}(\boldsymbol{\epsilon})\left[g\left(\boldsymbol{\epsilon}^{\prime}\right)-g_{0}\left(\boldsymbol{\epsilon}^{\prime}\right)\right] \delta(\Delta E+\boldsymbol{\epsilon}) \\
& +\int_{0}^{\infty} d \boldsymbol{\epsilon} \int_{0}^{\infty} d \boldsymbol{\epsilon}^{\prime} g_{0}\left(\boldsymbol{\epsilon}^{\prime}\right) \frac{1}{N} \sum_{p} n_{p}\left[g(\boldsymbol{\epsilon})-g_{1}(\boldsymbol{\epsilon})\right] \delta\left(\Delta-\boldsymbol{\epsilon}^{\prime}\right)+\int_{0}^{\infty} d \boldsymbol{\epsilon} \int_{0}^{\infty} d \epsilon^{\prime}\left[g\left(\boldsymbol{\epsilon}^{\prime}\right)-g_{0}\left(\boldsymbol{\epsilon}^{\prime}\right)\right] \frac{1}{N} \\
& \times \sum_{p} n_{p}\left[g(\boldsymbol{\epsilon})-g_{1}(\boldsymbol{\epsilon})\right] \delta(\Delta E) \\
= & \int_{\Delta E}^{\infty} d \epsilon g_{0}(\boldsymbol{\epsilon}) g_{1}(\boldsymbol{\epsilon}-\Delta E)+\left(1-P_{0}\right) g_{1}(-\Delta E) \theta(-\Delta E)-\left(1-P_{1}\right) g_{0}(\Delta E) \theta(\Delta E) \\
& +\left(1-P_{0}\right)\left(1-P_{1}\right) \delta(\Delta E) .
\end{aligned}
$$

The term with $\delta(\Delta E)$ is the term responsible for diffusive motion of the particles. Such a term does not actually make any contribution to the relaxation of the system and therefore we will not consider it from now on.

The probability distribution of accepted energy changes is given by

$$
P(\Delta E)=\frac{Q(\Delta E) W(\beta \Delta E)}{A},
$$

where $W(\beta \Delta E)$ is the Metropolis function

$$
W(\beta x)= \begin{cases}e^{-\beta x} & \text { if } x>0, \\ 1 & \text { if } x \leqslant 0 .\end{cases}
$$

The normalization factor is

$$
\begin{aligned}
A= & \int_{0}^{\infty} d \epsilon^{\prime} \int_{\epsilon^{\prime}}^{\infty} d \epsilon\left[e^{-\beta\left(\epsilon-\epsilon^{\prime}\right)} g_{0}(\epsilon) g_{1}\left(\epsilon^{\prime}\right)+g_{0}\left(\epsilon^{\prime}\right) g_{1}(\epsilon)\right] \\
& +\left(1-P_{0}\right) P_{1}+\left(1-P_{1}\right) \int_{0}^{\infty} d \epsilon g_{0}(\epsilon) e^{-\beta \epsilon}
\end{aligned}
$$

As $T \rightarrow 0$ the distribution $P$ becomes

$$
\begin{aligned}
P(\Delta E)= & \theta(-\Delta E) \\
& \times \frac{\int_{0}^{\infty} d \epsilon g_{0}(\epsilon) g_{1}(\epsilon-\Delta E)+\left(1-P_{0}\right) g_{1}(-\Delta E)}{A},
\end{aligned}
$$

with $A=\int_{0}^{\infty} d \epsilon^{\prime} \int_{\epsilon^{\prime}}^{\infty} d \epsilon g_{0}\left(\epsilon^{\prime}\right) g_{1}(\epsilon)+\left(1-P_{0}\right) P_{1}$.

The normalization factor $A$ is actually the acceptance rate of the Monte Carlo dynamics

$$
A=\int_{-\infty}^{\infty} d x W(\beta x) Q(x)
$$

as it was defined in Ref. [7].

Using the same notation we can write the energy evolution as

$$
\begin{aligned}
\frac{\partial E}{\partial t}= & \int_{-\infty}^{\infty} d x x W(\beta x) Q(x) \\
= & -P_{1} E-\int_{0}^{\infty} d \epsilon g_{1}(\epsilon) \epsilon+\int_{0}^{\infty} d \epsilon^{\prime} \int_{\epsilon^{\prime}}^{\infty} d \epsilon g_{1}\left(\epsilon^{\prime}\right) g_{0}(\epsilon) \\
& \times\left[e^{-\beta\left(\epsilon-\epsilon^{\prime}\right)}-1\right]\left(\epsilon-\epsilon^{\prime}\right) \\
& +\left(1-P_{1}\right) \int_{0}^{\infty} d \epsilon g_{0}(\epsilon) e^{-\beta \epsilon} \epsilon .
\end{aligned}
$$

The right-hand side of this equation can be equivalently obtained following the procedure presented in Appendix A. Indeed, by definition of energy density,

$$
\frac{\partial E}{\partial t}=-\int_{0}^{\infty} d \epsilon \epsilon \frac{\partial g_{0}(\epsilon)}{\partial t} .
$$

Inserting Eq. (24) in Eq. (D9) we get Eq. (D8) back.
[1] L. C. E. Struick, Physical Aging in Amorphous Polymers and Other Materials (Elsevier, Houston, 1978).

[2] J. P. Bouchaud, L. F. Cugliandolo, J. Kurchan, and M. Mezard, in Spin Glasses and Random Fields, edited by A. P. Young, Series on Directions in Condensed Matter Physics Vol. 12 (World Scientific, Singapore, 1998).

[3] W. Götze, Z. Phys. B: Condens. Matter 56, 139 (1984); W. Götze, in Liquids, Freezing and Glass Transition, edited by J.
P. Hansen, D. Levesque, and J. Zinn-Justin (North Holland, Amsterdam, 1991); W. Götze and L. Sjögren, Rep. Prog. Phys. 55, 241 (1992).

[4] A. V. Lopatin and L. B. Ioffe, Phys. Rev. B 60, 6412 (1999); Phys. Rev. Lett. 84, 4208 (2000).

[5] A. Crisanti, H. Horner, and H. J. Sommers, Z. Phys. B: Condens. Matter 92, 257 (1993).

[6] F. Ritort, Phys. Rev. Lett. 75, 1190 (1995). 
[7] L. L. Bonilla, F. G. Padilla, and F. Ritort, Physica A 250, 315 (1998).

[8] J. P. Bouchaud, J. Phys. I 4, 139 (1994); J. P. Bouchaud, A. Cometet, and C. Monthus, ibid. 5, 1521 (1995); C. Monthus and J. P. Bouchaud, J. Phys. A 29, 3847 (1996).

[9] G. H. Fredrickson and H. C. Andersen, Phys. Rev. Lett. 53, 1244 (1984); J. Jackle and S. Eisinger, Z. Phys. B: Condens. Matter 84, 115 (1991); M. Schulz and S. Trimper, Phys. Rev. B 53, 8421 (1996); W. Kob and H. C. Andersen, Phys. Rev. E 47, 3281 (1993).

[10] T. R. Kirkpatrick and D. Thirumalai, Phys. Rev. Lett. 58, 2091 (1987); T. R. Kirkpatrick, D. Thirumalai, and P. G. Wolynes, Phys. Rev. A 40, 1045 (1989).

[11] C. Dasgupta and S. Ramaswamy, Physica A 186, 314 (1992).

[12] W. Klein, H. Gould, R. Ramos, I. Clejan, and A. Melćuk, Physica A 205, 738 (1994).

[13] L. F. Cugliandolo and J. Kurchan, Phys. Rev. Lett. 71, 173 (1993).

[14] L. F. Cugliandolo, J. Kurchan, and L. Peliti, Phys. Rev. E 55, 3898 (1997); R. Exartier and L. Peliti, Eur. Phys. J.: Appl. Phys. 16, 119 (2000).

[15] S. Franz and F. Ritort, J. Phys. A 30, L359 (1997).

[16] Th. M. Nieuwenhuizen, J. Phys. A 31, L201 (1998); Phys. Rev. Lett. 80, 5580 (1998).

[17] Th. M. Nieuwenhuizen, Phys. Rev. E 61, 267 (2000).

[18] L. Leuzzi and Th. M. Nieuwenhuizen, Phys. Rev. E 64,
011508 (2001); 64, 066125 (2001).

[19] S. Franz and M. A. Virasoro, J. Phys. A 33, 891 (2000).

[20] C. Godrèche and J. M. Luck, J. Phys. A 29, 1915 (1996); 30, 6245 (1997); 32, 6033 (1999).

[21] A. Crisanti and F. Ritort, Philos. Mag. B 82, 143 (2002).

[22] S. Franz and F. Ritort, Europhys. Lett. 31, 507 (1995).

[23] J. P. Bouchaud, C. Godrèche, and M. Mézard, J. Phys. A 28, L603 (1995).

[24] A. Q. Tool, J. Am. Ceram. Soc. 29, 240 (1946).

[25] S. Franz and F. Ritort, J. Stat. Phys. 85, 131 (1996).

[26] H. Sompolinsky, Phys. Rev. Lett. 47, 935 (1981); H. Sompolinsky and A. Zippelius, Phys. Rev. B 25, 6860 (1982).

[27] L. Berthier and J.-L. Barrat, J. Chem. Phys. 116, 6228 (2002), and references therein.

[28] S. Fielding and P. Sollich, e-print cond-mat/0107627.

[29] A. Cavagna, I. Giardina, and G. Parisi, Phys. Rev. Lett. 83, 108 (1999).

[30] J. P. Bouchaud and D. S. Dean, J. Phys. I 5, 265 (1995).

[31] B. J. Kim, G. S. Jeon, and M. Y. Choi, Phys. Rev. Lett. 76, 4648 (1996).

[32] A. J. Brey, A. Prados, and B. Sanchez-Rey, Phys. Rev. B 55, 6343 (1997).

[33] Th. M. Nieuwenhuizen, J. Chem. Phys. 115, 8083 (2001).

[34] A. Garriga and F. Ritort, Eur. Phys. J. B 20, 105 (2001).

[35] L. Leuzzi and F. Ritort (unpublished). 